\title{
A central limit theorem for the overlap in the Hopfield model
}

\author{
Gentz, B
}

\begin{abstract}
We consider the Hopfield model with n neurons and an increasing number $p=p(n)$ of randomly chosen patterns. Under the condition $\left(p^{3} \log p\right) / n \rightarrow 0$, we prove for every fixed choice of overlap parameters a central limit theorem as $n \rightarrow \infty$, which holds for almost all realizations of the random patterns. In the special case where the temperature is above the critical one and there is no external magnetic field, the condition $\left(p^{2} \log p\right) / n \rightarrow 0$ suffices. As in the case of a finite number of patterns, the central limit theorem requires a centering which depends on the random patterns.
\end{abstract}

DOI: https://doi.org/10.1214/aop/1041903207

Posted at the Zurich Open Repository and Archive, University of Zurich

ZORA URL: https://doi.org/10.5167/uzh-22548

Journal Article

Originally published at:

Gentz, B (1996). A central limit theorem for the overlap in the Hopfield model. The Annals of Probability, 24(4):1809-1841.

DOI: https://doi.org/10.1214/aop/1041903207 


\title{
A CENTRAL LIMIT THEOREM FOR THE OVERLAP IN THE HOPFIELD MODEL ${ }^{1}$
}

\author{
BY BARBARA GENTZ
}

\section{Universität Zürich}

We consider the Hopfield model with $n$ neurons and an increasing number $p=p(n)$ of randomly chosen patterns. Under the condition $\left(p^{3} \log p\right) / n \rightarrow 0$, we prove for every fixed choice of overlap parameters a central limit theorem as $n \rightarrow \infty$, which holds for almost all realizations of the random patterns. In the special case where the temperature is above the critical one and there is no external magnetic field, the condition $\left(p^{2} \log p\right) / n \rightarrow 0$ suffices. As in the case of a finite number of patterns, the central limit theorem requires a centering which depends on the random patterns.

1. Introduction. The so-called Hopfield model was introduced as a model for an associative memory in the context of an artificial neural network with $n \in \mathbb{N}$ neurons. Roughly speaking, it deals with the problem of recognizing one out of $p \in \mathbb{N}$ stored patterns; for details, see [9], for example. We denote by $\xi^{\mu}=\left(\xi_{1}^{\mu}, \ldots, \xi_{n}^{\mu}\right) \in\{-1,1\}^{n}$ the codification of the $\mu$ th stored pattern, $\mu \in\{1, \ldots, p\}$, and by $\sigma_{i} \in\{-1,1\}$ the neural activity of the $i$ th neuron, $i \in\{1, \ldots, n\}$. Following Hopfield [10], we can describe the model as a spin system with the Hamiltonian

$$
H_{n}(\sigma, \xi)=-\frac{1}{2 n} \sum_{\mu=1}^{p} \sum_{i, j=1}^{n} \xi_{i}^{\mu} \xi_{j}^{\mu} \sigma_{i} \sigma_{j}, \quad n \in \mathbb{N},
$$

and the corresponding Glauber single-spin dynamics at zero temperature on the set of spin configurations $\{-1,1\}^{n}$. The spin system is said to retrieve the stored pattern $\xi^{\mu}$ when the spin configuration $\left(\sigma_{1}, \ldots, \sigma_{n}\right) \in\{-1,1\}^{n}$ converges under the Glauber dynamics to $\xi^{\mu}$, respectively, $-\xi^{\mu}$ for some $\mu \in$ $\{1, \ldots, p\}$.

In [1] generalized Glauber single-spin dynamics at finite temperature $1 / \beta \in$ $(0, \infty)$ are introduced on the set of spin configurations, again governed by the Hopfield Hamiltonian (1.1). These dynamics describe a reversible, irreducible Markov process which converges to its equilibrium distribution. The equilibrium distribution is the finite-volume Gibbs measure $P_{n, \beta, \xi}$ given by

$$
d P_{n, \beta, \xi}=\exp \left\{-\beta H_{n}(\sigma, \xi)\right\} d \mathbb{P}_{\sigma} / Z_{n, \beta, \xi},
$$

where $\mathbb{P}_{\sigma}=\left(\frac{1}{2} \delta_{-1}+\frac{1}{2} \delta_{1}\right)^{\otimes \mathbb{N}}$ and the partition function $Z_{n, \beta, \xi}$ is the appropriate normalization.

Received August 1995.

${ }^{1}$ Research supported by Swiss National Foundation Contract 21-298333.90.

AMS 1991 subject classifications. Primary 60F05; secondary 60K35, 82B44, 82C32.

Key words and phrases. Fluctuations, Hopfield model, overlap, neural networks, Laplace's method. 
From now on, we allow $p$ to depend on $n$ in a nondecreasing way satisfying $p \leq n$. We will consider $\left(\sigma_{i}\right)_{i \in \mathbb{N}}$ and $\left(\xi_{i}^{\mu}\right)_{i \in \mathbb{N}}$ with $\mu \in \mathbb{N}$ as random variables and assume that the family $\left\{\sigma_{i}, \xi_{j}^{\mu} \mid i, j, \mu \in \mathbb{N}\right\}$ is independent with $\mathbb{P}\left(\sigma_{i}=\right.$ $\pm 1)=1 / 2$ and $\mathbb{P}\left(\xi_{j}^{\mu}= \pm 1\right)=1 / 2$. We denote by $\mathbb{P}_{\xi}=\left(\frac{1}{2} \delta_{-1}+\frac{1}{2} \delta_{1}\right)^{\otimes \mathbb{N}^{2}}$ the marginal distribution of $\xi=\left(\xi_{i}^{\mu}\right)_{i, \mu \in \mathbb{N}}$, and similarly, by $\mathbb{P}_{\sigma}=\left(\frac{1}{2} \delta_{-1}+\frac{1}{2} \delta_{1}\right)^{\otimes \mathbb{N}}$ the marginal distribution of $\sigma=\left(\sigma_{i}\right)_{i \in \mathbb{N}}$.

In this setting, the Hopfield model may be regarded as an example of a mean-field spin system with random interaction. It has been studied widely; see [2] and the references given there. Note that already in 1977, Figotin and Pastur [6, 7] introduced and discussed a class of spin models which included the one with the Hopfield Hamiltonian (1.1).

For $i \in\{1, \ldots, n\}$ we denote by $\xi_{i}=\left(\xi_{i}^{\mu}\right)_{\mu \in\{1, \ldots, p\}}$ the vector consisting of the $i$ th components of the first $p$ patterns. Keep in mind that $\xi_{i} \in \mathbb{R}^{p}$ depends on $n$ via the dimension $p$. In the sequel, we will investigate the behavior of the so-called overlap

$$
\frac{1}{n} S_{n}(\sigma, \xi)=\frac{1}{n} \sum_{i=1}^{n} \xi_{i} \sigma_{i} \in \mathbb{R}^{p}
$$

under the equilibrium distribution $P_{n, \beta, \xi}$ as $n \rightarrow \infty$. The overlap compares the spin configuration $\sigma$ to the stored patterns $\xi^{\mu}, \mu \in\{1, \ldots, p\}$, in the sense that the $\mu$ th component of the overlap (1.3)—called the $\mu$ th overlap parameterequals 1 if and only if $\sigma_{i}=\xi_{i}^{\mu}$ for all $i \in\{1, \ldots, n\}$. The Hamiltonian (1.1) can be rewritten as a quadratic function of the overlap which provides the following convenient notation:

$$
H_{n}(\sigma, \xi)=-\frac{n}{2}\left\|\frac{1}{n} S_{n}(\sigma, \xi)\right\|^{2} .
$$

For simplicity, we will drop the explicit dependence on $\sigma$ and $\xi$ whenever no confusion may arise. So we will write $S_{n}$ instead of $S_{n}(\sigma, \xi)$, for instance.

For $p=1$ the Hopfield model is equivalent to the Curie-Weiss model. Let us fix some notation concerning the latter. For $\beta>0$, denote by $z^{ \pm}(\beta)$ the largest (resp. smallest) solution $z \in(-1,1)$ of the Curie-Weiss equation $\beta z=\operatorname{arctanh} z$. Note that $z^{+}(\beta)=-z^{-}(\beta) \neq 0$ for $\beta$ above the critical inverse temperature $\beta_{c}=1$, while $z^{ \pm}(\beta)=0$ for $\beta \leq \beta_{c}$. The free energy in the Curie-Weiss model equals

$$
f_{\mathrm{CW}}(\beta)=\frac{\beta}{2} z^{ \pm}(\beta)^{2}-I\left(z^{ \pm}(\beta)\right)
$$

where

$$
I(z)= \begin{cases}\frac{1}{2}(1+z) \log (1+z)+\frac{1}{2}(1-z) \log (1-z), & \text { for }|z| \leq 1 \\ \infty, & \text { otherwise }\end{cases}
$$

denotes the rate function which governs the large deviations of the spins per site in the Curie-Weiss model; compare [5], Section IV.4. To extend the definition of $z^{ \pm}(\beta)$ to the case of a so-called external magnetic field of strength 
$h \neq 0$, let $z(\beta, h)$ denote the unique solution $z \in(-1,1)$ of $\beta z+h=\operatorname{arctanh} z$ which satisfies $\operatorname{sign} z=\operatorname{sign} h$. In addition, we extend the notation $f_{\mathrm{CW}}(\beta)$ for the free energy in the Curie-Weiss model by defining

$$
f_{\mathrm{CW}}(\beta, h)= \begin{cases}\frac{\beta}{2} z(\beta, h)^{2}+h z(\beta, h)-I(z(\beta, h)), & \text { if } h \neq 0, \\ f_{\mathrm{CW}}(\beta), & \text { otherwise. }\end{cases}
$$

Note that

$$
f_{\mathrm{CW}}(\beta, h)=-\frac{1}{2 \beta}(\operatorname{arctanh} z-h)^{2}+\log \cosh \operatorname{arctanh} z,
$$

where $z=z^{ \pm}(\beta)$ in the case $h=0$ and $z=z(\beta, h)$, otherwise.

For investigating the behavior of the overlap (1.3), we also extend the notion of the Gibbs measure $P_{n, \beta, \xi}$ given in (1.2) to the case of an external magnetic field $h e_{l}$ with strength $h \neq 0$ in the direction of the $l$ th unit vector $e_{l}$ in $\mathbb{R}^{p}$. Let

$$
d P_{n, \beta, h e_{l}, \xi}=\exp \left\{-\beta H_{n}+\left\langle S_{n}, h e_{l}\right\rangle\right\} d \mathbb{P}_{\sigma} / Z_{n, \beta, h e_{l}, \xi},
$$

with the appropriate normalization $Z_{n, \beta, h e_{1}, \xi}$. In [2] it is shown that under the assumption $p / n \rightarrow 0$, for $\mathbb{P}_{\xi}$-almost all $\xi$, the distribution of the overlap $S_{n} / n$ under $P_{n, \beta, h e_{l}, \xi}$ converges weakly, as $n \rightarrow \infty$ and then $h \rightarrow 0 \pm$, to the Dirac measure concentrated at $z^{ \pm}(\beta) e_{l}$. Recall that $z^{ \pm}(\beta)=0$ for $\beta \leq \beta_{c}=1$, which implies uniqueness of the limiting measure in this case. Let us remark that under the assumption $p / n \rightarrow 0$, in [3] a large deviation principle is proved for the distribution of every finite choice of overlap parameters under the Gibbs measure (1.2).

Considering the above-mentioned weak convergence, it is natural to ask for the fluctuations of the overlap around $z^{ \pm}(\beta) e_{l}$. After some necessary preparations, we will state our main result, a central limit theorem for the overlap, in Section 2. In addition, we describe the asymptotic behavior of the partition functions in (1.2) and (1.5). In Section 3, we give the proofs of these results, based on a lemma that describes the asymptotic behavior of certain integrals. This key lemma itself is proved in Section 4 via an adaptation of Laplace's method.

2. Statement of results. To discuss the fluctuations of the overlap, we first choose a preferred pattern. This can be done in two different ways. In Theorem 2.5, we consider the unbiased Hamiltonian (1.1) and investigate the fluctuations under the condition that the overlap is already in a neighborhood of $z^{ \pm}(\beta) e_{l}$. Alternatively, the preferred pattern can be chosen by introducing an external magnetic field as in (1.5). The latter case is treated in Theorem 2.7. From now on, we will make the the following assumption.

GENERAL ASSUMPTION. Let $p=p(n)$ be a nondecreasing function of $n$ with $p \leq n$ for all $n \in \mathbb{N}$ and $\alpha=\alpha(n)=p / n \rightarrow 0$ as $n \rightarrow \infty$. 
Before stating the central limit theorem, we need to have a closer look at its centering. In the case of the unbiased Hamiltonian (1.1) with $\beta<\beta_{c}$, the central limit theorem holds with center zero. Otherwise, even in the case of bounded $p$, the central limit theorem for $(\beta, h) \neq\left(\beta_{\mathrm{c}}, 0\right)$ requires a $\xi$ dependent adjustment of the deterministic centering, where the deterministic one is suggested by the weak convergence of the overlap's distribution toward a Dirac measure. In [8], where the case of fixed $p$ is treated, a linear adjustment of the centering was chosen. Here we will choose the optimal centering instead of a linear approximation.

To describe the appropriate centering, we have to control the influence of the random patterns. This can be done via the $(p \times p)$ matrix $(1 / n) \sum_{i=1}^{n} \xi_{i} \xi_{i}^{\mathrm{T}}$, which will play a key role. Under the assumption $p / n \rightarrow 0$ this matrix approaches the $(p \times p)$ identity matrix as $n \rightarrow \infty$ in the sense that the operator norm of

$$
\Sigma^{n}(\xi)=\frac{1}{n} \sum_{i=1}^{n} \xi_{i} \xi_{i}^{\mathrm{T}}-\operatorname{Id}_{\mathbb{R}^{p}}
$$

converges to zero for $\mathbb{P}_{\xi}$-almost all $\xi$. This is the assertion of the following proposition, which is a modification of [2], Theorem 2.4 and mainly follows from the proof of that theorem; see Section 3.

Proposition 2.1. Fix $\varepsilon>0$ and define

$$
\alpha^{*}=\frac{1}{n} \max \left\{p,\left(\frac{3 \log n}{\log (1+\varepsilon)}\right)^{4}\right\} \quad \text { and } \quad \varepsilon_{n}=\sqrt{\alpha^{*}}\left(2+\sqrt{\alpha^{*}}\right)(1+\varepsilon) .
$$

Then there exists an $\tilde{n}_{0} \in \mathbb{N}$ such that

$$
\mathbb{P}_{\xi}\left(\left\|\Sigma^{n}(\xi)\right\|>\varepsilon_{n}\right) \leq \frac{1}{n^{2}}
$$

for all $n \geq \tilde{n}_{0}$. Furthermore, for $\mathbb{P}_{\xi^{-}}$almost all $\xi$, there exists an $n_{0}(\xi) \geq \tilde{n}_{0}$ such that

$$
\left\|\Sigma^{n}(\xi)\right\| \leq \varepsilon_{n}
$$

for all $n \geq n_{0}(\xi)$.

REMARK 2.2. In the case of bounded $p$, using the law of the iterated logarithm, the bound $\varepsilon_{n}$ in $(2.2)$ can be improved to $\sqrt{\alpha}(2 \log \log n)^{1 / 2}(1+\varepsilon)$; compare [8].

Let us fix some more notation. We denote by $e_{l}$ the $l$ th unit vector in $\mathbb{R}^{p}$. In addition, we set $e_{-l}=-e_{l}$ for convenience. The following index set $L$ will be used to describe those directions the overlap favors under the equilibrium measure. This index set depends on the dimension $p$, on the inverse temperature $\beta>0$, the presence $(h \neq 0)$ or absence $(h=0)$ of an external magnetic 
field and its direction $e_{l}$. Set

$$
L= \begin{cases}\{\operatorname{sign}(h) l\}, & \text { in the case } h \neq 0, \\ \{1\}, & \text { in the case } 0<\beta<\beta_{c} \text { and } h=0, \\ \{-p, \ldots,-1,1, \ldots, p\}, & \text { in the case } \beta>\beta_{c} \text { and } h=0 .\end{cases}
$$

We do not need to define the index set $L$ for $(\beta, h)=\left(\beta_{c}, 0\right)$ because the central limit theorem fails in this case; compare Remark 2.6(b). The following proposition is a first step toward defining the centering for the central limit theorem.

Proposition 2.3. Let $\beta>0$ and $h \geq 0$ be such that $(\beta, h) \neq\left(\beta_{c}, 0\right)$. As an abbreviation, we set $z=z^{+}(\beta)$ in the case $h=0$, and $z=z(\beta, h)$, otherwise. Let $l \in\{-p, \ldots,-1,1, \ldots, p\}$. For $\lambda \in \mathbb{R}^{p}$ define the $\xi$-dependent function

$$
\Phi(\lambda)=-\frac{1}{2 \beta}\left\|\lambda-h e_{l}\right\|^{2}+\frac{1}{n} \sum_{i=1}^{n} \log \cosh \left\langle\lambda, \xi_{i}\right\rangle .
$$

Then, for all strictly positive $c_{1}<\left(1-\beta\left(1-z^{2}\right)\right) / \beta$, there exists an $r_{1}>0$, depending on $\beta, h$ and $c_{1}$ only, and for $\mathbb{P}_{\xi}$-almost all $\xi$, there exists an $n_{1}(\xi) \geq$ $n_{0}(\xi)$, which does not depend on the choice of $l$, such that for all $n \geq n_{1}(\xi)$ the following assertions hold:

(a) For all $\lambda$ in the closed ball $\overline{B_{r_{1}}\left(\operatorname{arctanh} z e_{l}\right)}$, the matrix $-D^{2} \Phi(\lambda)$ is uniformly positive definite in the sense that

$$
\left\langle u,-D^{2} \Phi(\lambda) u\right\rangle \geq c_{1}\|u\|^{2} \text { for all } u \in \mathbb{R}^{p} .
$$

(b) On the set $\overline{B_{r_{1}}\left(\operatorname{arctanh} z e_{l}\right)}$, the map $\Phi$ has a unique maximum which is attained in a point $\lambda_{l}^{n}(\xi)$ satisfying

$$
\left\|\lambda_{l}^{n}(\xi)-\operatorname{arctanh} z e_{l}\right\| \leq c_{2} \varepsilon_{n},
$$

with $c_{2}=2|z| / c_{1}$. In particular, $\lambda_{l}^{n}(\xi)=0$ in the case $\beta<\beta_{c}$ and $h=0$.

REMARK 2.4. In [4], these assertions have been proved for $h=0$ and $p$ increasing at least linearly with $\log n$. The proof naturally extends to the case of small $p$ and $h>0$ and will be given in Section 3. If $n$ is a multiple of $2^{p}$ and the realization $\xi_{1}, \ldots, \xi_{n}$ takes all possible values with the same frequency, then $\lambda_{l}^{n}(\xi)=\operatorname{arctanh} z e_{l}$. Furthermore, due to (1.4), we always have $\Phi\left(\lambda_{l}^{n}(\xi)\right) \geq f_{\mathrm{CW}}(\beta, h)$.

From now on, we fix

$$
c_{1}=\frac{1}{2 \beta}\left(1-\beta\left(1-z^{2}\right)\right)
$$


for convenience. With the help of $\lambda_{l}^{n}(\xi)$, the appropriate centering $x_{l}^{n}(\xi)$ can be described by

$$
x_{l}^{n}(\xi)=\frac{1}{\beta}\left(\lambda_{l}^{n}(\xi)-h e_{l}\right)
$$

for $l \in\{-p, \ldots,-1,1, \ldots, p\}$. Since $\lambda_{l}^{n}(\xi)$ is only defined for $\mathbb{P}_{\xi}$-almost all $\xi$ and $n \geq n_{1}(\xi)$, we extend the definition of $x_{l}^{n}(\xi)$ by $x_{l}^{n}(\xi)=\beta^{-1}(\operatorname{arctanh} z-$ $h) e_{l}=z e_{l}$ whenever $\lambda_{l}^{n}(\xi)$ is not defined. Keep in mind that $x_{l}^{n}(\xi)$ depends on $\beta$ and $h$ although this is not indicated by the notation. By (2.4) and the Curie-Weiss equation, we always have

$$
\left\|x_{l}^{n}(\xi)-z e_{l}\right\| \leq \frac{1}{\beta} c_{2} \varepsilon_{n} .
$$

Now we are ready to state our main result, a $\mathbb{P}_{\xi}$-almost sure central limit theorem for the overlap. Let $\pi_{k}: \mathbb{R}^{p} \rightarrow \mathbb{R}^{k}$ (with $k \leq p$ ) denote the canonical projection.

THEOREM 2.5. Let $\beta>0, \beta \neq \beta_{c}, h=0$ and let $k \in \mathbb{N}$ satisfy $k \leq p$ in the case of bounded $p$. Fix $\varepsilon \in\left(0, z^{+}(\beta) / 8\right)$ in the case $\beta>\beta_{c}$ and $\varepsilon>0$ arbitrary, otherwise. Suppose that $\left(p^{2} \log p\right) / n \rightarrow 0$ in the case $\beta<\beta_{c}$ and $\left(p^{3} \log p\right) / n \rightarrow 0$, otherwise. Then, for every $l \in L$ and $\mathbb{P}_{\xi^{-}}$-almost all $\xi$, the measures

$$
P_{n, \beta, \xi}\left(\sqrt{n} \pi_{k}\left[\frac{S_{n}}{n}-x_{l}^{n}(\xi)\right] \in \cdot \mid\left\|\frac{S_{n}}{n}-z^{+}(\beta) e_{l}\right\|<\varepsilon\right)
$$

converge weakly, as $n$ tends to infinity, to the Gaussian distribution on $\mathbb{R}^{k}$ with mean zero and covariance matrix

$$
C_{\beta}=\frac{1-z^{+}(\beta)^{2}}{1-\beta\left(1-z^{+}(\beta)^{2}\right)} \operatorname{Id}_{\mathbb{R}^{k}} .
$$

REMARK 2.6. (a) The condition $\varepsilon<z^{+}(\beta) / 8$ in the case $\beta>\beta_{c}$ especially assures that the balls of radius $\varepsilon$ with centers $z^{+}(\beta) e_{\nu}, \nu \in L$, are disjoint.

(b) Recall that the fluctuations of the spin per site in the Curie-Weiss model, which corresponds to a single component of the overlap in the Hopfield model, are non-Gaussian at the critical inverse temperature $\beta_{c}=1$ (cf. [5], Theorem V.9.5).

(c) For $\beta<\beta_{c}$, the theorem reduces to

$$
P_{n, \beta, \xi}\left(\pi_{k}\left[\frac{S_{n}}{\sqrt{n}}\right] \in \cdot \mid\left\|\frac{S_{n}}{n}\right\|<\varepsilon\right) \Rightarrow \mathscr{N}\left(0, \frac{1}{1-\beta} \operatorname{Id}_{\mathbb{R}^{k}}\right),
$$

$\mathbb{P}_{\xi}$-almost surely, which yields

$$
P_{n, \beta, \xi}\left(\pi_{k}\left[\frac{S_{n}}{\sqrt{n}}\right]\right)^{-1} \Rightarrow \mathscr{N}\left(0, \frac{1}{1-\beta} \operatorname{Id}_{\mathbb{R}^{k}}\right)
$$

as an immediate consequence of Remark 2.10(b). 
(d) By the triangle inequality, it is obvious that the conditioning in (2.6) may be replaced by $\left\|S_{n} / n-x_{l}^{n}(\xi)\right\|<\varepsilon$. In addition, it is intuitively clear that the conditioning can as well be replaced by $\left\|\pi_{k}\left[S_{n} / n-z^{+}(\beta) e_{l}\right]\right\|<\varepsilon$ or $\left\|\pi_{k}\left[S_{n} / n-x_{l}^{n}(\xi)\right]\right\|<\varepsilon$, respectively. Indeed, this can be shown along the lines of the proof of the central limit theorem given in Section 3.

Selecting the preferred pattern by an external magnetic field yields the following version of the central limit theorem.

THEOREM 2.7. Suppose that $\left(p^{3} \log p\right) / n \rightarrow 0$. Let $\beta>0, h>0, l \in \mathbb{Z} \backslash\{0\}$ and $k \in \mathbb{N}$. In the case of bounded $p$, let $|l| \leq p$ and $k \leq p$ in addition. Then, for $\mathbb{P}_{\xi^{-}}$almost all $\xi$, the measures

$$
P_{n, \beta, h e_{l}, \xi}\left(\sqrt{n} \pi_{k}\left[\frac{S_{n}}{n}-x_{l}^{n}(\xi)\right]\right)^{-1}
$$

converge weakly, as $n$ tends to infinity, to the Gaussian distribution on $\mathbb{R}^{k}$ with mean zero and covariance matrix

$$
C_{\beta, h}=\frac{1-z(\beta, h)^{2}}{1-\beta\left(1-z(\beta, h)^{2}\right)} \operatorname{Id}_{\mathbb{R}^{k}} .
$$

REMARK 2.8. The central limit theorems formulated in Theorems 2.5 and 2.7 remain valid when the centering $x_{l}^{n}(\xi)$ is replaced by

$$
\left[\operatorname{Id}_{\mathbb{R}^{p}}+\frac{1}{1-\beta\left(1-z^{2}\right)} \Sigma^{n}\right] z e_{l}
$$

with $z=z^{+}(\beta)$ in the case $h=0$ and $z=z(\beta, h)$, otherwise. This is the linear adjustment of the deterministic centering $z e_{l}$ which was chosen in [8] in the case of fixed $p$.

Before turning to the proofs of Theorems 2.5 and 2.7, we will state a result on the asymptotic behavior of the partition function and the probability that the overlap is in a small neighborhood of $z^{+}(\beta) e_{l}$ or $z(\beta, h) e_{l}$, respectively. It is of interest on its own and follows directly from Lemma 3.3.

Theorem 2.9. (a) Let $\beta>0, \beta \neq \beta_{c}$ and $h=0$. Fix $\varepsilon \in\left(0, z^{+}(\beta) / 8\right)$ in the case $\beta>\beta_{c}$ and $\varepsilon>0$ arbitrary, otherwise. Then, under the condition $\left(p^{2} \log p\right) / n \rightarrow 0$, for every $l \in L$ and for $\mathbb{P}_{\xi^{-}}$almost all $\xi$,

$$
\begin{aligned}
& P_{n, \beta, \xi}\left(\left\|\frac{S_{n}}{n}-z^{+}(\beta) e_{l}\right\|<\varepsilon\right) \\
& \quad=\frac{1}{Z_{n, \beta, \xi}} \frac{\exp \left\{n \Phi\left(\lambda_{l}^{n}(\xi)\right)\right\}}{\sqrt{\operatorname{det}\left(-\beta D^{2} \Phi\left(\lambda_{l}^{n}(\xi)\right)\right)}}[1+o(1)] \exp \left\{\mathscr{O}\left(p \delta_{n}\right)\right\}
\end{aligned}
$$

and

$$
Z_{n, \beta, \xi}=\left(\sum_{\nu \in L} \frac{\exp \left\{n \Phi\left(\lambda_{\nu}^{n}(\xi)\right)\right\}}{\sqrt{\operatorname{det}\left(-\beta D^{2} \Phi\left(\lambda_{\nu}^{n}(\xi)\right)\right)}}\right)[1+o(1)] \exp \left\{\mathscr{O}\left(p \delta_{n}\right)\right\}
$$

as $n \rightarrow \infty$. The properties of the sequence $\left\{\delta_{n}\right\}_{n \in \mathbb{N}}$ will be specified below. 
(b) Let $\beta>0$ and $h>0$. Assume that $l \in \mathbb{Z} \backslash\{0\}$ satisfies $|l| \leq p$ in the case of bounded $p$. Then, under the condition $\left(p^{2} \log p\right) / n \rightarrow 0$, for every $\varepsilon>0$ and for $\mathbb{P}_{\xi^{-}}$almost all $\xi$,

$$
P_{n, \beta, h e_{l}, \xi}\left(\left\|\frac{S_{n}}{n}-z(\beta, h) e_{l}\right\|<\varepsilon\right)=[1+o(1)] \exp \left\{\mathscr{O}\left(p \delta_{n}\right)\right\}
$$

and

$$
Z_{n, \beta, h e_{l}, \xi}=\frac{\exp \left\{n \Phi\left(\lambda_{l}^{n}(\xi)\right)\right\}}{\sqrt{\operatorname{det}\left(-\beta D^{2} \Phi\left(\lambda_{l}^{n}(\xi)\right)\right)}}[1+o(1)] \exp \left\{\mathscr{O}\left(p \delta_{n}\right)\right\}
$$

as $n \rightarrow \infty$.

The sequence $\left\{\delta_{n}\right\}_{n \in \mathbb{N}}$ in (a) and (b) tends to zero as $n \rightarrow \infty$. In the special case $z=0$ (i.e., $\beta<\beta_{c}$ and $h=0$ ), $p \delta_{n} \rightarrow 0$ holds. If the stronger assumption $\left(p^{3} \log p\right) / n \rightarrow 0$ holds, then $p \delta_{n} \rightarrow 0$ is always satisfied.

REMARK 2.10. (a) The error terms of the form $o(1)$ in the above statement depend on $\xi$ in the following way: given $\varrho>0$, there exists for $\mathbb{P}_{\xi}$-almost all $\xi$ an $n(\xi, \varrho)$ such that the corresponding error term is bounded by $\varrho$ for all $n \geq n(\xi, \varrho)$. The error terms of the form $\mathscr{O}\left(p \delta_{n}\right)$ depend on $\xi$ in the sense that there exist a constant $C>0$ and an $n(\xi, C)$ such that the corresponding error term is bounded by $C p \delta_{n}$ for all $n \geq n(\xi, C)$.

(b) For $\beta<\beta_{c}$ and $h=0$ the theorem states that

$$
P_{n, \beta, \xi}\left(\left\|\frac{S_{n}}{n}\right\|<\varepsilon\right)=1+o(1)
$$

and

$$
Z_{n, \beta, \xi}=(1-\beta)^{-p / 2}[1+o(1)]
$$

$\mathbb{P}_{\xi}$-almost surely as $n \rightarrow \infty$.

3. Proof of the central limit theorem. We start by proving Proposition 2.1, which controls the influence of the random patterns. The proof relies heavily on the Appendix to [2], where the same problem is considered. In [2], the accent is put on the rate of decay as $n \rightarrow \infty$ of the probability that the norm of the random matrix in question exceeds the given bound $\sqrt{\alpha}(2+\sqrt{\alpha})(1+\varepsilon)$, while in Proposition 2.1, we aim for less restrictive conditions on $p$ such that the bound $\sqrt{\alpha}(2+\sqrt{\alpha})(1+\varepsilon)$ still holds $\mathbb{P}_{\xi}$-almost surely.

Proof of Proposition 2.1. First, we consider the case of large $p$. In [2], Lemma A.1, the following bound on the expectation of the trace of powers of $\Sigma^{n}(\xi)$ is given: there exists a constant $C>0$ such that for all $k \in \mathbb{N}$ satisfying $k^{6} \leq n$ and $k^{4} \leq p$,

$$
\mathbb{E}_{\xi} \operatorname{tr}\left[\Sigma^{n}(\xi)\right]^{k} \leq \operatorname{Cn}[\sqrt{\alpha}(2+\sqrt{\alpha})]^{k+1} .
$$


By Chebyshev's inequality, for even $k$, we have

$$
\begin{aligned}
\mathbb{P}_{\xi}\left(\left\|\Sigma^{n}(\xi)\right\|>\sqrt{\alpha}(2+\sqrt{\alpha})(1+\varepsilon)\right) & \leq[\sqrt{\alpha}(2+\sqrt{\alpha})(1+\varepsilon)]^{-k} \mathbb{E}_{\xi} \operatorname{tr}\left[\Sigma^{n}(\xi)\right]^{k} \\
& \leq C n \sqrt{\alpha}(2+\sqrt{\alpha})(1+\varepsilon)^{-k}
\end{aligned}
$$

For $p \geq(3 \log n / \log (1+\varepsilon))^{4}$ and large enough $n$ we may choose an even $k$ satisfying $[3 \log n+\log (3 C)+\log \sqrt{\alpha}] / \log (1+\varepsilon) \leq k \leq p^{1 / 4}$ because $\log \sqrt{\alpha} \rightarrow$ $-\infty$. Hence, in this case,

$$
\mathbb{P}_{\xi}\left(\left\|\Sigma^{n}(\xi)\right\|>\sqrt{\alpha}(2+\sqrt{\alpha})(1+\varepsilon)\right) \leq \frac{1}{n^{2}} .
$$

To treat the case $p<(3 \log n / \log (1+\varepsilon))^{4}$, just note that for fixed $n$ the operator norm of the $(p \times p)$ matrix $\Sigma^{n}(\xi)$ is a nondecreasing function of the dimension $p$. Therefore, (2.1) follows directly from (3.1) for all $p$. Since the right-hand side of (2.1) is summable over $n,(2.2)$ follows by an application of the Borel-Cantelli lemma.

Before turning to the proofs of Proposition 2.3, the central limit theorem and Theorem 2.9, we need to investigate the map $\Phi$ defined in (2.3). For this purpose, we need the following lemma, which is mainly taken from a preliminary version of [4]. It will serve as an important tool when estimating $(1 / n) \sum_{i=1}^{n}\left\langle v, \xi_{i}\right\rangle^{2}$ because it gives uniform bounds for the crucial part of the sum when $\left|\left\langle v, \xi_{i}\right\rangle\right|$ exceeds a given bound $a>0$ although $\|v\| \leq \varrho$ is small.

LEMMA 3.1. There exists a constant $c_{3}>0$ such that for all $\varrho>0$ and $a>0$, which both may depend on $n$, and for $\mathbb{P}_{\xi^{-}}$-almost all $\xi$, there exists an $n_{2}(\xi) \geq n_{0}(\xi)$ such that for all $n \geq n_{2}(\xi)$,

$$
\frac{1}{n} \sum_{i=1}^{n}\left\langle v, \xi_{i}\right\rangle^{2} 1_{\left\{\left|\left\langle v, \xi_{i}\right\rangle\right|>a\right\}} \leq \Gamma(n, a / \varrho)\|v\|^{2}
$$

for all $v \in \mathbb{R}^{p}$ with $\|v\| \leq \varrho$, where

$$
\Gamma(n, a)=8\left[\alpha|\log \alpha|+c_{3} \alpha+4 \exp \left\{-\frac{a^{2}}{8}\right\}+\frac{2}{n} \log n\right] .
$$

Since in [4] the proof of the analogous lemma is given only for $p$ increasing at least linearly with $\log n$, we will show that it naturally extends to the case of small $p$.

Proof of Lemma 3.1. For $v \in \mathbb{R}^{p}$ set

$$
X_{a}(v)=\frac{1}{n} \sum_{i=1}^{n}\left\langle v, \xi_{i}\right\rangle^{2} 1_{\left\{\left|\left\langle v, \xi_{i}\right\rangle\right|>a\right\}} .
$$

Although we are interested in $X_{a}(v)$ only, we need to consider the related variables

$$
Y_{a}(v)=\frac{1}{n} \sum_{i=1}^{n} 1_{\left\{\left|\left\langle v, \xi_{i}\right\rangle\right|>a\right\}}
$$


in addition. For fixed $v \neq 0$, we have by Chebyshev's inequality

$$
\begin{aligned}
\mathbb{P}_{\xi}\left(X_{a}(v) \geq x\right) & \leq \exp \{-t x n\}\left(\mathbb{E}_{\xi}\left(\exp \left\{t\left\langle v, \xi_{1}\right\rangle^{2} 1_{\left\{\left|\left\langle v, \xi_{1}\right\rangle\right|>a\right\}}\right\}\right)\right)^{n} \\
& \leq \exp \{-t x n\}\left(1+\mathbb{E}_{\xi}\left(\exp \left\{t\left\langle v, \xi_{1}\right\rangle^{2}\right\} 1_{\left\{\left|\left\langle v, \xi_{1}\right\rangle\right|>a\right\}}\right)\right)^{n}
\end{aligned}
$$

for all $x>0$ and every $t>0$. Using the estimate $\cosh y \leq \exp \left\{y^{2} / 2\right\}$,

$$
\mathbb{P}_{\xi}\left(\left\langle v, \xi_{1}\right\rangle>a\right) \leq \exp \{-s a\} \mathbb{E}_{\xi} \exp \left\{s\left\langle v, \xi_{1}\right\rangle\right\} \leq \exp \{-s a\} \exp \left\{s^{2}\|v\|^{2} / 2\right\}
$$

follows for every $s>0$, and therefore, choosing $s=a /\|v\|^{2}$ yields

$$
\mathbb{P}_{\xi}\left(\left\langle v, \xi_{1}\right\rangle>a\right) \leq \exp \left\{-a^{2} /\left(2\|v\|^{2}\right)\right\} .
$$

By partial integration,

$$
\begin{aligned}
& \mathbb{E}_{\xi}\left(\exp \left\{t\left\langle v, \xi_{1}\right\rangle^{2}\right\} 1_{\left\{\left|\left\langle v, \xi_{1}\right\rangle\right|>a\right\}}\right) \\
& \quad=2 \int_{(a, \infty)} \exp \left\{t x^{2}\right\} \mathbb{P}_{\xi}\left(\left\langle v, \xi_{1}\right\rangle\right)^{-1}(d x) \\
& \quad=2 \exp \left\{t a^{2}\right\} \mathbb{P}_{\xi}\left(\left\langle v, \xi_{1}\right\rangle>a\right)+2 \int_{(a, \infty)} 2 t x \exp \left\{t x^{2}\right\} \mathbb{P}_{\xi}\left(\left\langle v, \xi_{1}\right\rangle>x\right) d x .
\end{aligned}
$$

From (3.3), we find for all $t<\left(2\|v\|^{2}\right)^{-1}$,

$$
\begin{aligned}
& \mathbb{E}_{\xi}\left(\exp \left\{t\left\langle v, \xi_{1}\right\rangle^{2}\right\} 1_{\left\{\left|\left\langle v, \xi_{1}\right\rangle\right|>a\right\}}\right) \\
& \quad \leq 2 \exp \left\{t a^{2}\right\} \exp \left\{\frac{-a^{2}}{2\|v\|^{2}}\right\}+2 \int_{a}^{\infty} 2 t x \exp \left\{t x^{2}\right\} \exp \left\{\frac{-x^{2}}{2\|v\|^{2}}\right\} d x \\
& \quad=\frac{2}{1-2 t\|v\|^{2}} \exp \left\{-a^{2}\left(\frac{1}{2\|v\|^{2}}-t\right)\right\} .
\end{aligned}
$$

Choosing $t=\left(4\|v\|^{2}\right)^{-1}$, from (3.2) it follows that

$$
\mathbb{P}_{\xi}\left(X_{a}(v) \geq x\right) \leq \exp \left\{-n \frac{x}{4\|v\|^{2}}\right\}\left(1+4 \exp \left\{-\frac{a^{2}}{4\|v\|^{2}}\right\}\right)^{n} .
$$

Another application of Chebyshev's inequality and (3.3) gives

$$
\mathbb{P}_{\xi}\left(Y_{a}(v) \geq x\right) \leq \exp \{-t x n\}\left(1+2 \exp \{t\} \exp \left\{-\frac{a^{2}}{2\|v\|^{2}}\right\}\right)^{n}
$$

for all $x>0$ and every $t>0$, and the choice $t=a^{2} /\left(4\|v\|^{2}\right)$ yields

$$
\mathbb{P}_{\xi}\left(Y_{a}(v) \geq x\right) \leq \exp \left\{-n \frac{a^{2} x}{4\|v\|^{2}}\right\}\left(1+2 \exp \left\{-\frac{a^{2}}{4\|v\|^{2}}\right\}\right)^{n} .
$$

Instead of a fixed $v$, we finally want to consider $v$ taken from a ball in $\mathbb{R}^{p}$, where we are aiming for uniform bounds. As a first step in this direction, we 
will consider $v$ from a finite set. Denote by $\mathscr{W}_{p, r}$ the cubic lattice in $\mathbb{R}^{p}$ with spacing $r / \sqrt{p}$, and for $r<r_{0}$ define $\mathscr{W}_{p, r}\left(r_{0}\right)=\mathscr{W}_{p, r} \cap \overline{B_{r_{0}}(0)}$. Then there exists a constant $c>0$ such that

$$
\left|\mathscr{W}_{p, r}\left(r_{0}\right)\right| \leq \exp \left\{p\left(\log \left(r_{0} / r\right)+c\right)\right\} .
$$

This estimate allows us to estimate $X_{a}(v)$ and $Y_{a}(v)$ uniformly on the grid $\mathscr{W}_{p, r}\left(r_{0}\right)$. By (3.4),

$$
\begin{aligned}
& \mathbb{P}_{\xi}\left(\sup _{v \in \mathscr{H}_{p, r}\left(r_{0}\right)} X_{a}(v) \geq 4 r_{0}^{2}\left[\alpha\left(\log \frac{r_{0}}{r}+c\right)+4 \exp \left\{-\frac{a^{2}}{4 r_{0}^{2}}\right\}+\frac{2}{n} \log n\right]\right) \\
& \quad \leq \frac{1}{n^{2}}
\end{aligned}
$$

and, similarly for each $k \in \mathbb{N}$ by (3.5),

$$
\mathbb{P}_{\xi}\left(\sup _{v \in \mathscr{H}_{p, r}\left(r_{0}\right)} Y_{a}(v) \geq 4 \frac{r_{0}^{2}}{a^{2}}\left[\alpha\left(\log \frac{r_{0}}{r}+c\right)+2+\frac{2}{n} \log (n k)\right]\right) \leq \frac{1}{(n k)^{2}}
$$

Instead of taking the supremum over the grid $\mathscr{W}_{p, r}\left(r_{0}\right)$, we want to consider the supremum over all $v \in \overline{B_{r_{0}}(0)}$. For this purpose, we will introduce a sequence of lattices $\mathscr{W}_{p, r_{k}}, k \in \mathbb{N}_{0}$, with $r_{0}$ and $r_{k} \searrow 0$ specified below. This allows us to rewrite each $v \in \overline{B_{r_{0}}(0)}$ for arbitrary $k_{0} \in \mathbb{N}$ as

$$
v=\sum_{k=1}^{k_{0}} v_{k}+v_{k_{0}+1}
$$

with $v_{k} \in \mathscr{W}_{p, r_{k}}\left(r_{k-1}\right)$ for $1 \leq k \leq k_{0}$ and $\left\|v_{k_{0}+1}\right\| \leq r_{k_{0}}$. Such a representation will be used together with the following estimates on $X_{a}(v)$ and $Y_{a}(v)$. For $a_{1}, \widetilde{a}>0$ such that $a=a_{1}+\tilde{a}$ and for arbitrary vectors $v, v_{1}, \widetilde{v} \in \mathbb{R}^{p}$ with $v=v_{1}+\widetilde{v}$ we have

$$
\begin{aligned}
X_{a}(v) & \leq 2 X_{a_{1}}\left(v_{1}\right)+\frac{2}{n} \sum_{i=1}^{n}\left\langle v_{1}, \xi_{i}\right\rangle^{2} 1_{\left\{\left|\left\langle v, \xi_{i}\right\rangle\right|>a\right\}} 1_{\left\{\left|\left\langle v_{1}, \xi_{i}\right\rangle\right| \leq a_{1}\right\}}+\frac{2}{n} \sum_{i=1}^{n}\left\langle\widetilde{v}, \xi_{i}\right\rangle^{2} \\
& \leq 2 X_{a_{1}}\left(v_{1}\right)+2 a_{1}^{2} Y_{\widetilde{a}}(\widetilde{v})+2\left\langle\widetilde{v}, \frac{1}{n} \sum_{i=1}^{n} \xi_{i} \xi_{i}^{\mathrm{T}} \widetilde{v}\right\rangle
\end{aligned}
$$

and

$$
Y_{a}(v) \leq Y_{a_{1}}\left(v_{1}\right)+Y_{\widetilde{a}}(\widetilde{v}) .
$$

Note that the bound (3.10) on $X_{a}$ is weaker than the one given in [4]. This weaker bound will suffice for our purpose.

Next, we choose $r_{k}=\alpha^{k}$ for defining the lattice $\mathscr{W}_{p, r_{k}}, k \in \mathbb{N}_{0}$, and $a_{1}=$ $a / \sqrt{2}$. Set $\gamma=1-1 / \sqrt{2}$ as an abbreviation. By (3.10) with $v_{1} \in \mathscr{W}_{p, r_{1}}\left(r_{0}\right)$ and the estimates (3.7) and (2.1), we find for arbitrary $y>0$ and all $n \geq \widetilde{n}_{0}$ with 
$\widetilde{n}_{0}$ from Proposition 2.1,

$$
\begin{aligned}
\mathbb{P}_{\xi}\left(\sup _{\|v\| \leq 1} X_{a}(v) \geq 8\left[\alpha\left(\left|\log r_{1}\right|+c\right)+\right.\right. & \left.4 \exp \left\{-\frac{a_{1}^{2}}{4}\right\}+\frac{2}{n} \log n\right] \\
& \left.+2 a_{1}^{2} y+2 r_{1}^{2}\left(1+\varepsilon_{n}\right)\right) \\
\leq & \frac{1}{n^{2}}+\mathbb{P}_{\xi}\left(\sup _{\|\widetilde{v}\| \leq r_{1}} Y_{\gamma a}(\widetilde{v}) \geq y\right)+\mathbb{P}_{\xi}\left(\left\|\frac{1}{n} \sum_{i=1}^{n} \xi_{i} \xi_{i}^{T}\right\| \geq 1+\varepsilon_{n}\right) \\
\leq & \frac{2}{n^{2}}+\mathbb{P}_{\xi}\left(\sup _{\|\widetilde{v}\| \leq r_{1}} Y_{\gamma a}(\widetilde{v}) \geq y\right) .
\end{aligned}
$$

It remains to choose an appropriate $y$ and to estimate the last probability in (3.12) afterward. For this purpose, we choose a decreasing sequence $\left(d_{k}\right)_{k \in \mathbb{N}}$ by setting $d_{k}=\gamma \alpha 2^{-(k-1)}$ for $k \in \mathbb{N}$. Let $n$ be large enough to satisfy $2 \alpha<1$. Then there exists a $k_{0}=k_{0}(n) \geq 2$ such that $d_{k_{0}} \geq \sqrt{p} r_{k_{0}}$ and, therefore $Y_{d_{k_{0}}}(w)=0$ for all $\|w\| \leq r_{k_{0}}$. By the estimate (3.11) and the representation (3.9),

$$
\sup _{\|\widetilde{v}\| \leq r_{1}} Y_{\gamma a}(\widetilde{v}) \leq \sum_{k=2}^{k_{0}} \sup _{v_{k} \in \mathscr{H}_{p, r_{k}}\left(r_{k-1}\right)} Y_{d_{k}}\left(v_{k}\right)
$$

follows. Since $\alpha \leq 1 / 3$ for large enough $n$,

$$
\sum_{k=2}^{\infty} 4\left(\frac{r_{k-1}}{d_{k}}\right)^{2}\left[\alpha\left(\log \frac{r_{k-1}}{r_{k}}+c\right)+2+\frac{2}{n} \log (n k)\right] \leq \widetilde{c}_{3} \frac{\alpha^{2}}{a^{2}}
$$

holds for these $n$ with some constant $\widetilde{c}_{3}>0$. From (3.8) and (3.13) it follows that

$$
\mathbb{P}_{\xi}\left(\sup _{\|\widetilde{v}\| \leq r_{1}} Y_{\gamma a}(\widetilde{v}) \geq \widetilde{c}_{3} \frac{\alpha^{2}}{a^{2}}\right) \leq \sum_{k=2}^{\infty} \frac{1}{(n k)^{2}} \leq \frac{1}{n^{2}} \frac{\pi^{2}}{6}
$$

and, therefore, returning to (3.12), we find for all $n \geq \widetilde{n}_{0}$ with $\alpha \leq 1 / 3$,

$$
\mathbb{P}_{\xi}\left(\sup _{\|v\| \leq 1} X_{a}(v) \geq \Gamma(n, a)\right) \leq\left(2+\frac{\pi^{2}}{6}\right) \frac{1}{n^{2}} .
$$

An application of the Borel-Cantelli lemma shows the existence of an $n_{2}(\xi)$ such that

$$
\sup _{\|v\| \leq 1} X_{a}(v) \leq \Gamma(n, a)
$$

for $\mathbb{P}_{\xi}$-almost all $\xi$ and all $n \geq n_{2}(\xi)$. Applying (3.14) with $v$ replaced by the normalized vector $v /\|v\|$ for $v \in \overline{B_{\varrho}(0)} \backslash\{0\}$ yields

$$
X_{a}(v) \leq \sup _{\|\widetilde{v}\| \leq 1} X_{a / \varrho}(\widetilde{v})\|v\|^{2} \leq \Gamma(n, a / \varrho)\|v\|^{2}
$$

for all $v \in \mathbb{R}^{p}$ with $\|v\| \leq \varrho$. 
As a first application of the preceding lemma, we can now prove the existence of a unique maximum of $\Phi$ in a small neighborhood of $\operatorname{arctanh} z e_{l}$, where $z$ is defined as in Proposition 2.3. The case $h=0$ and $p$ increasing at least linearly with $\log n$ has been proved in [4] by showing local convexity of $-\Phi$. The proof naturally extends to the case $h \neq 0$.

Proof of Proposition 2.3. First note that

$$
D^{2} \Phi(\lambda)=-\frac{1}{\beta} \operatorname{Id}_{\mathbb{R}^{p}}+\frac{1}{n} \sum_{i=1}^{n} \cosh ^{-2}\left(\left\langle\lambda, \xi_{i}\right\rangle\right) \xi_{i} \xi_{i}^{\mathrm{T}} .
$$

To prove that $-D^{2} \Phi(\lambda)$ is positive definite for $\lambda$ in a neighborhood of $\operatorname{arctanh} z e_{l}$, we choose $u, v \in \mathbb{R}^{p} \backslash\{0\}$ arbitrarily. Then

$$
\begin{aligned}
\langle u,- & \left.D^{2} \Phi\left(\operatorname{arctanh} z e_{l}+v\right) u\right\rangle \\
= & \frac{1}{\beta}\|u\|^{2}-\frac{1}{n} \sum_{i=1}^{n}\left(1-z^{2}\right)\left\langle u, \xi_{i} \xi_{i}^{\mathrm{T}} u\right\rangle \\
- & \frac{1}{n} \sum_{i=1}^{n}\left[\cosh ^{-2}\left(\operatorname{arctanh} z\left\langle e_{l}, \xi_{i}\right\rangle+\left\langle v, \xi_{i}\right\rangle\right)\right. \\
& \left.-\cosh ^{-2}\left(\operatorname{arctanh} z\left\langle e_{l}, \xi_{i}\right\rangle\right)\right]\left\langle u, \xi_{i}\right\rangle^{2},
\end{aligned}
$$

where we used $\cosh ^{-2} \operatorname{arctanh} z=1-z^{2}$. Applying the estimate

$$
\left|\cosh ^{-2}(x+y)-\cosh ^{-2}(x)\right| \leq \min \{1,2|y|\},
$$

the bound from Proposition 2.1 on the random matrix and Lemma 3.1, we find that for every auxiliary $a>0, \mathbb{P}_{\xi}$-almost all $\xi$ and all $n \geq n_{2}(\xi)$,

$$
\begin{aligned}
\langle u,- & \left.D^{2} \Phi\left(\operatorname{arctanh} z e_{l}+v\right) u\right\rangle \\
\geq & {\left[\frac{1}{\beta}\left(1-\beta\left(1-z^{2}\right)\left(1+\varepsilon_{n}\right)\right)\right]\|u\|^{2} } \\
& -\frac{2}{n} \sum_{i=1}^{n}\left|\left\langle v, \xi_{i}\right\rangle\right|\left\langle u, \xi_{i}\right\rangle^{2} 1_{\left\{\left|\left\langle v, \xi_{i}\right\rangle\right| \leq a\|v\|\right\}}-\frac{1}{n} \sum_{i=1}^{n}\left\langle u, \xi_{i}\right\rangle^{2} 1_{\left\{\left|\left\langle v, \xi_{i}\right\rangle\right|>a\|v\|\right\}} \\
\geq & {\left[\frac{1}{\beta}\left(1-\beta\left(1-z^{2}\right)\left(1+\varepsilon_{n}\right)\right)-2 a\|v\|\left(1+\varepsilon_{n}\right)\right]\|u\|^{2} } \\
& -\frac{1}{n} \sum_{i=1}^{n} \max \left\{\left\langle\frac{u}{\|u\|}, \xi_{i}\right\rangle^{2} 1_{\left\{\left|\left\langle u, \xi_{i}\right\rangle\right|>a\|u\|\right\}},\left\langle\frac{v}{\|v\|}, \xi_{i}\right\rangle^{2} 1_{\left\{\left|\left\langle v, \xi_{i}\right\rangle\right|>a\|v\|\right\}}\right\}\|u\|^{2} \\
\geq & {\left[\frac{1}{\beta}\left(1-\beta\left(1-z^{2}\right)\left(1+\varepsilon_{n}\right)\right)-2 a\|v\|\left(1+\varepsilon_{n}\right)-2 \Gamma(n, a)\right]\|u\|^{2} . }
\end{aligned}
$$


Choosing $n$ and $a$ large makes $\Gamma(n, a)$ arbitrarily small and $\varepsilon_{n}$ tends to zero as $n \rightarrow \infty$. Therefore, there exist $a>0$ and $\tilde{n}_{1} \in \mathbb{N}$ such that

$$
\left(1-z^{2}\right) \varepsilon_{n}+2 \Gamma(n, a) \leq \frac{1}{2}\left[\frac{1}{\beta}\left(1-\beta\left(1-z^{2}\right)\right)-c_{1}\right]
$$

for all $n \geq \widetilde{n}_{1}$. Having fixed $a$, there exists an $r_{1}$ such that

$$
2 a\|v\|\left(1+\varepsilon_{n}\right) \leq \frac{1}{2}\left[\frac{1}{\beta}\left(1-\beta\left(1-z^{2}\right)\right)-c_{1}\right]
$$

holds for all $n \geq \widetilde{n}_{1}$ and all $v$ satisfying $\|v\| \leq r_{1}$. This proves

$$
\left\langle u,-D^{2} \Phi\left(\operatorname{arctanh} z e_{l}+v\right) u\right\rangle \geq c_{1}\|u\|^{2}
$$

for $\mathbb{P}_{\xi}$-almost all $\xi$, all $u \in \mathbb{R}^{p},\|v\| \leq r_{1}$ and all $n \geq n_{1}(\xi)=\max \left\{n_{2}(\xi), \tilde{n}_{1}\right\}$.

Next, we will show that $\Phi$ does not attain its maximum on the boundary of $B_{r_{1}}\left(\operatorname{arctanh} z e_{l}\right)$. By Taylor's formula, for all $v \in \overline{B_{r_{1}}\left(\operatorname{arctanh} z e_{l}\right)}$, there exists a $\theta \in[0,1]$ such that

$$
\begin{aligned}
& \Phi\left(\operatorname{arctanh} z e_{l}+v\right) \\
& \quad=\Phi\left(\operatorname{arctanh} z e_{l}\right)+\left\langle\Sigma^{n}(\xi) z e_{l}, v\right\rangle-\frac{1}{2}\left\langle v,-D^{2} \Phi\left(\operatorname{arctanh} z e_{l}+\theta v\right) v\right\rangle \\
& \quad \leq \Phi\left(\operatorname{arctanh} z e_{l}\right)+|z| \varepsilon_{n}\|v\|-\frac{c_{1}}{2}\|v\|^{2}
\end{aligned}
$$

for all $n \geq n_{1}(\xi)$ by our bound on $\Sigma^{n}(\xi)$ and the estimate (3.17). Therefore,

$$
\Phi\left(\operatorname{arctanh} z e_{l}+v\right)<\Phi\left(\operatorname{arctanh} z e_{l}\right)
$$

for all $v$ satisfying $c_{2} \varepsilon_{n}<\|v\| \leq r_{1}$ with $c_{2}=2|z| / c_{1}$. This establishes the existence of a unique maximum of $\Phi$ on the set $\overline{B_{r_{1}}\left(\operatorname{arctanh} z e_{l}\right)}$ as well as (2.4).

As an abbreviation, for every $\nu \in L$, set

$$
C_{\nu}^{n}(\xi)=-D^{2} \Phi\left(\lambda_{\nu}^{n}(\xi)\right)=\frac{1}{\beta} \operatorname{Id}_{\mathbb{R}^{p}}-\frac{1}{n} \sum_{i=1}^{n} \cosh ^{-2}\left(\left\langle\lambda_{\nu}^{n}(\xi), \xi_{i}\right\rangle\right) \xi_{i} \xi_{i}^{\mathrm{T}} .
$$

The following lemma shows that $C_{\nu}^{n}(\xi)$ converges to a deterministic diagonal matrix provided that $\sqrt{p} \varepsilon_{n} \rightarrow 0$.

LEMMA 3.2. Let $\beta>0$ and $h \in \mathbb{R}$ such that $(\beta, h) \neq\left(\beta_{c}, 0\right)$. Choose an $l \in \mathbb{Z} \backslash\{0\}$, satisfying $|l| \leq p$ in the case of bounded $p$. Then there exists a constant $c_{4}>0$ such that

$$
\sup _{\nu \in L}\left\|C_{\nu}^{n}(\xi)-\frac{1}{\beta}\left[1-\beta\left(1-z^{2}\right)\right] \operatorname{Id}_{\mathbb{R}^{p}}\right\| \leq c_{4} \sqrt{p} \varepsilon_{n}
$$

for $\mathbb{P}_{\xi}$-almost all $\xi$ and all $n \geq n_{1}(\xi)$. 
Proof. By the identity $\cosh ^{-2} \operatorname{arctanh} z=1-z^{2}$, (3.16), (2.4) and Proposition 2.1, for $\mathbb{P}_{\xi}$-almost all $\xi$, all $n \geq n_{1}(\xi)$ and all $\nu \in L$,

$$
\begin{aligned}
\| C_{\nu}^{n}(\xi) & -\frac{1}{\beta}\left[1-\beta\left(1-z^{2}\right)\right] \operatorname{Id}_{\mathbb{R}^{p}} \| \\
\leq & \left\|\frac{1}{n} \sum_{i=1}^{n}\left[\cosh ^{-2}\left(\left\langle\lambda_{\nu}^{n}(\xi), \xi_{i}\right\rangle\right)-\cosh ^{-2}\left(\left\langle\operatorname{arctanh} z e_{\nu}, \xi_{i}\right\rangle\right)\right] \xi_{i} \xi_{i}^{\mathrm{T}}\right\| \\
& +\left(1-z^{2}\right)\left\|\frac{1}{n} \sum_{i=1}^{n} \xi_{i} \xi_{i}^{\mathrm{T}}-\mathrm{Id}_{\mathbb{R}^{p}}\right\| \\
\leq & 2\left\|\lambda_{\nu}^{n}(\xi)-\operatorname{arctanh} z e_{\nu}\right\| \sqrt{p}\left\|\frac{1}{n} \sum_{i=1}^{n} \xi_{i} \xi_{i}^{\mathrm{T}}\right\|+\left\|\Sigma^{n}\right\| \\
\leq & {\left[2 c_{2} \sqrt{p}\left(1+\varepsilon_{n}\right)+1\right] \varepsilon_{n} . }
\end{aligned}
$$

The proof of the central limit theorem as well as the proof of Theorem 2.9 is based on the following lemma, which describes the asymptotic behavior of certain integrals. We will give the proof in Section 4.

LEMMA 3.3. Let $\beta>0$ and $h \in \mathbb{R}$ be such that $(\beta, h) \neq\left(\beta_{c}, 0\right)$, and let $l \in \mathbb{Z} \backslash\{0\}, y \in \mathbb{R}^{k}$ and $k \geq|l|$. Assume $k \leq p$ in the case of bounded $p$. As an abbreviation, set

$$
y_{n}=\frac{1}{\sqrt{n}}\left(\begin{array}{l}
y \\
0
\end{array}\right) \in \mathbb{R}^{p} .
$$

For all $\lambda \in \mathbb{R}^{p}$, define the $\xi$-dependent function $\Psi$ by

$$
\Psi(\lambda)=-\frac{1}{2 \beta}\left\|\lambda-h e_{l}-y_{n}\right\|^{2}+\frac{1}{n} \sum_{i=1}^{n} \log \cosh \left\langle\lambda, \xi_{i}\right\rangle .
$$

Then there exist $R_{0}, r_{0}>0$, depending on $\beta, h$ and $y$ only, such that for all $R \geq R_{0}$, all $r \leq r_{0}$ and $\mathbb{P}_{\xi^{-}}$almost all $\xi$ the following assertions hold:

(a) Under the general assumption $p / n \rightarrow 0$,

$$
\int_{\bigcap_{v \in L} B_{r}^{\mathrm{c}}\left(\operatorname{arctanh} z e_{\nu}\right)} \exp \{n \Psi(\lambda)\} d \lambda=o(1) \int_{\mathbb{R}^{p}} \exp \{n \Psi(\lambda)\} d \lambda
$$

as $n \rightarrow \infty$.

(b) Again under the general assumption $p / n \rightarrow 0$, for every $\nu \in L$,

$$
\int_{B_{r}\left(\operatorname{arctanh} z e_{\nu}\right)} \exp \{n \Psi(\lambda)\} d \lambda=[1+o(1)] \int_{B_{R \varepsilon_{n}}\left(\operatorname{arctanh} z e_{\nu}\right)} \exp \{n \Psi(\lambda)\} d \lambda
$$

and

$$
\begin{aligned}
& \int_{\bigcup_{\nu \in L} B_{r}\left(\operatorname{arctanh} z e_{\nu}\right)} \exp \{n \Psi(\lambda)\} d \lambda \\
& \quad=[1+o(1)] \int_{\bigcup_{\nu \in L} B_{R \varepsilon_{n}}\left(\operatorname{arctanh} z e_{\nu}\right)} \exp \{n \Psi(\lambda)\} d \lambda
\end{aligned}
$$

as $n \rightarrow \infty$. 
(c) Furthermore, under the condition $\left(p^{2} \log p\right) / n \rightarrow 0$, for every $\nu \in L$,

$$
\begin{aligned}
\left(\frac{n}{2 \pi \beta}\right)^{p / 2} \int_{B_{R \varepsilon_{n}}\left(\operatorname{arctanh} z e_{\nu}\right)} \exp \{n \Psi(\lambda)\} d \lambda \\
=\operatorname{det}\left(\beta C_{\nu}^{n}(\xi)\right)^{-1 / 2} \exp \left\{n\left[\Phi\left(\lambda_{\nu}^{n}(\xi)\right)+\left\langle x_{\nu}^{n}(\xi), y_{n}\right\rangle\right]\right\} \\
\quad \times \exp \left\{-\frac{n}{2 \beta}\left\langle y_{n},\left[\operatorname{Id}_{\mathbb{R}^{p}}-\frac{1}{\beta} C_{\nu}^{n}(\xi)^{-1}\right] y_{n}\right\rangle\right\} \\
\quad \times[1+o(1)] \exp \left\{\mathscr{O}\left(p \delta_{n}\right)\right\}
\end{aligned}
$$

and

$$
\begin{aligned}
& \left(\frac{n}{2 \pi \beta}\right)^{p / 2} \int_{\mathbb{R}^{p}} \exp \{n \Psi(\lambda)\} d \lambda \\
& =\left(\sum_{\nu \in L} \operatorname{det}\left(\beta C_{\nu}^{n}(\xi)\right)^{-1 / 2} \exp \left\{n\left[\Phi\left(\lambda_{\nu}^{n}(\xi)\right)+\left\langle x_{\nu}^{n}(\xi), y_{n}\right\rangle\right]\right\}\right. \\
& \left.\quad \times \exp \left\{-\frac{n}{2 \beta}\left\langle y_{n},\left[\operatorname{Id}_{\mathbb{R}^{p}}-\frac{1}{\beta} C_{\nu}^{n}(\xi)^{-1}\right] y_{n}\right\rangle\right\}\right) \\
& \quad \times[1+o(1)] \exp \left\{\mathscr{O}\left(p \delta_{n}\right)\right\}
\end{aligned}
$$

as $n \rightarrow \infty$ with a sequence $\left\{\delta_{n}\right\}_{n \in \mathbb{N}}$ tending to zero, which has the same properties as the one in Theorem 2.9 .

The following lemma will be used to replace the indicator function of a ball by its smoothed version obtained via the convolution with the continuous density of a Gaussian random variable.

Lemma 3.4. Let $\beta>0, n, p \in \mathbb{N}$ and $\varepsilon>0$. Then for all $x \in \mathbb{R}^{p}$,

$$
\begin{aligned}
& \left(\frac{n \beta}{2 \pi}\right)^{p / 2} \int_{\mathbb{R}^{p}} 1_{B_{\varepsilon / 2}(0)}(x+\lambda) \exp \left\{-\frac{n \beta}{2}\|\lambda\|^{2}\right\} d \lambda-2^{p / 2} \exp \left\{-\frac{n \beta}{16} \varepsilon^{2}\right\} \\
& \leq 1_{B_{\varepsilon}(0)}(x) \\
& \quad \leq\left(\frac{n \beta}{2 \pi}\right)^{p / 2} \int_{\mathbb{R}^{p}} 1_{B_{2 \varepsilon}(0)}(x+\lambda) \exp \left\{-\frac{n \beta}{2}\|\lambda\|^{2}\right\} d \lambda+2^{p / 2} \exp \left\{-\frac{n \beta}{4} \varepsilon^{2}\right\} .
\end{aligned}
$$


Proof. By splitting the domain of integration into the two sets with $\|\lambda\|<$ $\varepsilon / 2$ and $\|\lambda\| \geq \varepsilon / 2$, we find

$$
\begin{aligned}
& \left(\frac{n \beta}{2 \pi}\right)^{p / 2} \int_{\mathbb{R}^{p}} 1_{B_{\varepsilon / 2}(0)}(x+\lambda) \exp \left\{-\frac{n \beta}{2}\|\lambda\|^{2}\right\} d \lambda \\
& \leq\left(\frac{n \beta}{2 \pi}\right)^{p / 2}\left[1_{B_{\varepsilon}(0)}(x) \int_{\mathbb{R}^{p}} \exp \left\{-\frac{n \beta}{2}\|\lambda\|^{2}\right\} d \lambda\right. \\
& \left.\quad+\int_{\mathbb{R}^{p}} 1_{B_{\varepsilon / 2}^{\mathrm{c}}(0)}(\lambda) \exp \left\{-\frac{n \beta}{2}\|\lambda\|^{2}\right\} d \lambda\right] \\
& \leq 1_{B_{\varepsilon}(0)}(x)+\exp \left\{-\frac{n \beta}{4}\left(\frac{\varepsilon}{2}\right)^{2}\right\}\left(\frac{n \beta}{2 \pi}\right)^{p / 2} \int_{\mathbb{R}^{p}} \exp \left\{-\frac{n \beta}{4}\|\lambda\|^{2}\right\} d \lambda \\
& =1_{B_{\varepsilon}(0)}(x)+2^{p / 2} \exp \left\{-\frac{n \beta}{4}\left(\frac{\varepsilon}{2}\right)^{2}\right\} .
\end{aligned}
$$

Similarly,

$$
\begin{aligned}
& \left(\frac{n \beta}{2 \pi}\right)^{p / 2} \int_{\mathbb{R}^{p}} 1_{B_{2 \varepsilon}(0)}(x+\lambda) \exp \left\{-\frac{n \beta}{2}\|\lambda\|^{2}\right\} d \lambda \\
& \geq\left(\frac{n \beta}{2 \pi}\right)^{p / 2} 1_{B_{\varepsilon}(0)}(x) \int_{\mathbb{R}^{p}} 1_{B_{\varepsilon}(0)}(\lambda) \exp \left\{-\frac{n \beta}{2}\|\lambda\|^{2}\right\} d \lambda \\
& \quad=1_{B_{\varepsilon}(0)}(x)\left[1-\left(\frac{n \beta}{2 \pi}\right)^{p / 2} \int_{\mathbb{R}^{p}} 1_{B_{\varepsilon}^{\mathrm{c}}(0)}(\lambda) \exp \left\{-\frac{n \beta}{2}\|\lambda\|^{2}\right\} d \lambda\right] \\
& \geq 1_{B_{\varepsilon}(0)}(x)-2^{p / 2} \exp \left\{-\frac{n \beta}{4} \varepsilon^{2}\right\} .
\end{aligned}
$$

Now, we are ready to give the proof of the central limit theorem. Let us start with the case of the unbiased Hamiltonian.

Proof OF THEOREM 2.5. First note that we may restrict ourselves to the case $k \geq|l|$. Furthermore, we may assume that $p \geq k$. By [12], Lemma C1, it suffices to show for $\mathbb{P}_{\xi}$-almost all $\xi$ the pointwise convergence of the Laplace transforms of

$$
Q_{n}=P_{n, \beta, \xi}\left(\sqrt{n} \pi_{k}\left[\frac{S_{n}}{n}-x_{l}^{n}(\xi)\right] \in \cdot \mid\left\|\frac{S_{n}}{n}-z e_{l}\right\|<\varepsilon\right)
$$

[with $z=z^{+}(\beta)$ ] to the Laplace transform of the Gaussian measure on $\mathbb{R}^{k}$ with mean zero and covariance matrix $C_{\beta}$. For this purpose, let $y \in \mathbb{R}^{k}$ and define $y_{n}=(1 / \sqrt{n})\left(\begin{array}{l}y \\ 0\end{array}\right) \in \mathbb{R}^{p}$ as in Lemma 3.3. Then the Laplace transform $Q_{n}^{*}(y)$ of $Q_{n}$ at $y$ equals

$$
Q_{n}^{*}(y)=\zeta_{n, \varepsilon} \int \exp \left\{n\left\langle\frac{S_{n}}{n}-x_{l}^{n}(\xi), y_{n}\right\rangle\right\} 1_{\left\{\left\|\left(S_{n} / n\right)-z e_{l}\right\|<\varepsilon\right\}} d P_{n, \beta, \xi},
$$


where

$$
\zeta_{n, \varepsilon}=\left[P_{n, \beta, \xi}\left(\left\|\frac{S_{n}}{n}-z e_{l}\right\|<\varepsilon\right)\right]^{-1} .
$$

With the help of Lemma 3.4, the Laplace transform $Q_{n}^{*}(y)$ can be estimated from above, respectively, below by

$$
\begin{aligned}
& \frac{1}{Z_{n, \beta, \xi}} \zeta_{n, \varepsilon}\left(\frac{n \beta}{2 \pi}\right)^{p / 2} \\
& \quad \times \iint_{\mathbb{R}^{p}} 1_{B_{2^{ \pm 1}}(0)}\left(\frac{S_{n}}{n}-z e_{l}+\lambda\right) \\
& \quad \times \exp \left\{n\left[-\frac{\beta}{2}\|\lambda\|^{2}+\frac{\beta}{2}\left\|\frac{S_{n}}{n}\right\|^{2}+\left\langle\frac{S_{n}}{n}-x_{l}^{n}(\xi), y_{n}\right\rangle\right]\right\} d \lambda d \mathbb{P}_{\sigma} \\
& \pm \zeta_{n, \varepsilon} 2^{p / 2} \exp \left\{-\frac{n \beta}{16} \varepsilon^{2}+2 \sqrt{n k}\|y\|\right\},
\end{aligned}
$$

where we applied

$$
\left|\left\langle\frac{S_{n}}{n}-x_{l}^{n}(\xi), y_{n}\right\rangle\right| \leq 2 \sqrt{\frac{k}{n}}\|y\| .
$$

Substituting $(1 / \beta)\left(\lambda-y_{n}\right)-(1 / n) S_{n}$ for $\lambda$ and using $p / n \rightarrow 0$, we see that $Q_{n}^{*}(y)$ is bounded by

$$
\begin{aligned}
& \frac{1}{Z_{n, \beta, \xi}} \zeta_{n, \varepsilon}\left(\frac{n}{2 \pi \beta}\right)^{p / 2} \exp \left\{-n\left\langle x_{l}^{n}(\xi), y_{n}\right\rangle\right\} \\
& \quad \times \int_{\left\{\left\|\lambda-\beta z e_{l}\right\|<2^{ \pm 1} \beta \varepsilon \pm\left\|y_{n}\right\|\right\}} \int \exp \left\{n\left[-\frac{1}{2 \beta}\left\|\lambda-y_{n}\right\|^{2}+\left\langle\frac{S_{n}}{n}, \lambda\right\rangle\right]\right\} d \mathbb{P}_{\sigma} d \lambda \\
& \quad \pm \zeta_{n, \varepsilon} \exp \left\{-c_{5} n\right\}
\end{aligned}
$$

with some constant $c_{5}>0$. Let $n$ be large enough to satisfy $\left\|y_{n}\right\| \leq \beta \varepsilon / 4$. Performing the integration with respect to $\mathbb{P}_{\sigma}$ (cf. [8], for instance) and using the Curie-Weiss equation $\beta z=\operatorname{arctanh} z$ shows that $Q_{n}^{*}(y)$ is bounded by

$$
\begin{aligned}
& \frac{1}{Z_{n, \beta, \xi}} \zeta_{n, \varepsilon}\left(\frac{n}{2 \pi \beta}\right)^{p / 2} \exp \left\{-n\left\langle x_{l}^{n}(\xi), y_{n}\right\rangle\right\} \\
& \quad \times \int_{\left\{\left\|\lambda-\operatorname{arctanh} z e_{l}\right\|<2^{ \pm 2} \beta \varepsilon\right\}} \exp \left\{n\left[-\frac{1}{2 \beta}\left\|\lambda-y_{n}\right\|^{2}+\frac{1}{n} \sum_{i=1}^{n} \log \cosh \left\langle\lambda, \xi_{i}\right\rangle\right]\right\} d \lambda \\
& \quad \pm \zeta_{n, \varepsilon} \exp \left\{-c_{5} n\right\} .
\end{aligned}
$$

Applying Lemma 3.3(b), (c) and Theorem 2.9(a) yields

$$
\begin{aligned}
Q_{n}^{*}(y)= & \exp \left\{-\frac{n}{2 \beta}\left\langle y_{n},\left[\operatorname{Id}_{\mathbb{R}^{p}}-\frac{1}{\beta} C_{l}^{n}(\xi)^{-1}\right] y_{n}\right)\right\}[1+o(1)] \\
& +\mathscr{O}\left(\zeta_{n, \varepsilon} \exp \left\{-c_{5} n\right\}\right) .
\end{aligned}
$$


It remains to investigate the error term. By Theorem 2.9,

$$
\zeta_{n, \varepsilon}=\sum_{\nu \in L}\left(\frac{\operatorname{det}\left(\beta C_{\nu}^{n}(\xi)\right)}{\operatorname{det}\left(\beta C_{l}^{n}(\xi)\right)}\right)^{-1 / 2} \exp \left\{n\left[\Phi\left(\lambda_{\nu}^{n}(\xi)\right)-\Phi\left(\lambda_{l}^{n}(\xi)\right)\right]\right\}[1+o(1)] .
$$

Lemma 3.2 implies

$$
\left(\frac{\operatorname{det}\left(\beta C_{\nu}^{n}(\xi)\right)}{\operatorname{det}\left(\beta C_{l}^{n}(\xi)\right)}\right)^{-1 / 2} \leq\left(1+\mathscr{O}\left(\sqrt{p} \varepsilon_{n}\right)\right)^{p},
$$

where the error term $\mathscr{O}\left(\sqrt{p} \varepsilon_{n}\right)$ does not depend on $\nu \in L$. Furthermore, we know that $\Phi\left(\operatorname{arctanh} z e_{\nu}\right)=f_{\mathrm{CW}}(\beta)$ does not depend on $\nu$. By the definition of $\lambda_{\nu}^{n}(\xi)$ as a local maximum of $\Phi$ [see (2.4)], (3.15) and Proposition 2.1, we find for $\mathbb{P}_{\xi}$-almost all $\xi$,

$$
\begin{aligned}
& \left|\Phi\left(\lambda_{\nu}^{n}(\xi)\right)-\Phi\left(\lambda_{l}^{n}(\xi)\right)\right| \\
& \quad \leq 2 \max _{\mu \in L}\left|\Phi\left(\lambda_{\mu}^{n}(\xi)\right)-\Phi\left(\operatorname{arctanh} z e_{\mu}\right)\right| \\
& \quad \leq \max _{\mu \in L} \max \left\{\left\|D^{2} \Phi(\lambda)\right\|\left\|\lambda_{\mu}^{n}(\xi)-\operatorname{arctanh} z e_{\mu}\right\|^{2} \mid \lambda \in B_{c_{2} \varepsilon_{n}}\left(\operatorname{arctanh} z e_{\mu}\right)\right\} \\
& \quad \leq\left(\frac{1}{\beta}+1+\varepsilon_{n}\right)\left(c_{2} \varepsilon_{n}\right)^{2}=\mathscr{O}\left(\varepsilon_{n}^{2}\right) .
\end{aligned}
$$

Therefore,

$$
\begin{aligned}
\zeta_{n, \varepsilon} & \leq 2 p\left(1+\mathscr{O}\left(\sqrt{p} \varepsilon_{n}\right)\right)^{p} \exp \left\{n \mathscr{O}\left(\varepsilon_{n}^{2}\right)\right\} \\
& =2 \exp \{\log p+o(p)+o(n)\}=2 \exp \{o(n)\},
\end{aligned}
$$

which implies, by (3.20) and Lemma 3.2,

$$
\begin{aligned}
Q_{n}^{*}(y) & =\exp \left\{-\frac{n}{2 \beta}\left\langle y_{n},\left[\operatorname{Id}_{\mathbb{R}^{p}}-\frac{1}{\beta} C_{l}^{n}(\xi)^{-1}\right] y_{n}\right\rangle\right\}[1+o(1)]+o(1) \\
& \rightarrow \exp \left\{\frac{1}{2}\left\langle y, C_{\beta} y\right\rangle\right\} \quad \text { as } n \rightarrow \infty .
\end{aligned}
$$

Since $y \mapsto \exp \left\{\frac{1}{2}\left\langle y, C_{\beta} y\right\rangle\right\}$ is the Laplace transform of the Gaussian distribution with mean zero and covariance matrix $C_{\beta}$, the proof is complete.

Next we give the proof of the central limit theorem in the case of an external magnetic field.

Proof of TheOREM 2.7. As in the preceding proof, we will show for $\mathbb{P}_{\xi^{-}}$ almost all $\xi$ the pointwise convergence of the Laplace transforms $Q_{n}^{*}$ of the measures

$$
Q_{n}=P_{n, \beta, h e_{l}, \xi}\left(\sqrt{n} \pi_{k}\left[\frac{S_{n}}{n}-x_{l}^{n}(\xi)\right]\right)^{-1}
$$


As before, we may assume $k \geq|l|$. Let again $y \in \mathbb{R}^{k}$ and $y_{n}=(1 / \sqrt{n})\left(\begin{array}{l}y \\ 0\end{array}\right) \in \mathbb{R}^{p}$. Then the Laplace transform $Q_{n}^{*}(y)$ of $Q_{n}$ at $y$ equals

$$
\begin{aligned}
Q_{n}^{*}(y)=\frac{1}{Z_{n, \beta, h e_{l}, \xi}} \int & \exp \left\{n\left\langle\frac{S_{n}}{n}-x_{l}^{n}(\xi), y_{n}\right\rangle\right\} \\
& \times \exp \left\{\frac{n \beta}{2}\left\|\frac{S_{n}}{n}\right\|^{2}+n\left\langle\frac{S_{n}}{n}, h e_{l}\right\rangle\right\} d \mathbb{P}_{\sigma},
\end{aligned}
$$

and by a Gaussian integration,

$$
\begin{aligned}
Q_{n}^{*}(y)= & \frac{1}{Z_{n, \beta, h e_{l}, \xi}}\left(\frac{n}{2 \pi \beta}\right)^{p / 2} \exp \left\{-n\left\langle x_{l}^{n}(\xi), y_{n}\right\rangle\right\} \\
& \times \int_{\mathbb{R}^{p}} \int \exp \left\{n\left[-\frac{1}{2 \beta}\left\|\lambda-h e_{l}-y_{n}\right\|^{2}+\left\langle\frac{S_{n}}{n}, \lambda\right\rangle\right]\right\} d \mathbb{P}_{\sigma} d \lambda .
\end{aligned}
$$

Performing the integration with respect to $\mathbb{P}_{\sigma}$ as before,

$$
\begin{aligned}
Q_{n}^{*}(y)= & \frac{1}{Z_{n, \beta, h e_{l}, \xi}}\left(\frac{n}{2 \pi \beta}\right)^{p / 2} \exp \left\{-n\left\langle x_{l}^{n}(\xi), y_{n}\right\rangle\right\} \\
& \times \int_{\mathbb{R}^{p}} \exp \left\{n\left[-\frac{1}{2 \beta}\left\|\lambda-h e_{l}-y_{n}\right\|^{2}+\frac{1}{n} \sum_{i=1}^{n} \log \cosh \left\langle\lambda, \xi_{i}\right\rangle\right]\right\} d \lambda .
\end{aligned}
$$

Applying Lemma 3.3(c) and Theorem 2.9(b), we find

$$
Q_{n}^{*}(y)=\exp \left\{-\frac{n}{2 \beta}\left\langle y_{n},\left[\operatorname{Id}_{\mathbb{R}^{p}}-\frac{1}{\beta} C_{\nu}^{n}(\xi)^{-1}\right] y_{n}\right)\right\}[1+o(1)]
$$

and, therefore,

$$
Q_{n}^{*}(y) \rightarrow \exp \left\{\frac{1}{2}\left\langle y, C_{\beta, h} y\right\rangle\right\} \quad \text { as } n \rightarrow \infty
$$

by Lemma 3.2, which proves the central limit theorem.

Proof of Theorem 2.9. The proof of (2.7) and (2.9) follows from Lemma 3.3 (with $y=0$ ) with the help of Lemma 3.4 in the same way as the central limit theorem in the case of the unbiased Hamiltonian is proved, and the identities (2.8) and (2.10) can be proved via Lemma 3.3 in the same way as the central limit theorem in the case of an external magnetic field.

4. Proof of Lemma 3.3. The whole section is devoted to the proof of Lemma 3.3. Recall that we want to investigate the asymptotic behavior of integrals of the type

$$
\left(\frac{n}{2 \pi \beta}\right)^{p / 2} \int_{\mathbb{R}^{p}} \exp \{n \Psi(\lambda)\} d \lambda,
$$


where $\Psi$ is given by (3.19). Considering $y_{n}=(1 / \sqrt{n})\left(\begin{array}{l}y \\ 0\end{array}\right)$ as an error term, Proposition 2.3 suggests the following way of splitting the domain of integration into three parts: the outer region

$$
U_{r}=\left(\bigcup_{\nu \in L} B_{r}\left(\operatorname{arctanh} z e_{\nu}\right)\right)^{c}
$$

the intermediate region

$$
V_{r, R}=U_{r}^{c} \backslash \bigcup_{\nu \in L} B_{R \varepsilon_{n}}\left(\operatorname{arctanh} z e_{\nu}\right)
$$

and the inner region

$$
W_{R}=\bigcup_{\nu \in L} B_{R \varepsilon_{n}}\left(\operatorname{arctanh} z e_{\nu}\right)
$$

We will choose $r$ sufficiently small and $R$ sufficiently large, while $\varepsilon_{n}$ stems from Proposition 2.1. Remember that $\Psi=\Phi$ in the case $y=0$ and that $\Phi$ attains a local maximum in $\overline{B_{c_{2} \varepsilon_{n}}\left(\operatorname{arctanh} z e_{\nu}\right)}$ for each $\nu \in L$.

Outer region. First, we will show that the outer region does not contribute to the asymptotic behavior of (4.1). The estimate

$$
\log \cosh x \leq \frac{1}{4 \beta} x^{2}+\max _{t \in \mathbb{R}}\left\{-\frac{1}{4 \beta} t^{2}+\log \cosh t\right\}=\frac{1}{4 \beta} x^{2}+f_{\mathrm{CW}}(2 \beta)
$$

and the bound on the random matrix given in Proposition 2.1 show that for $\mathbb{P}_{\xi}$-almost all $\xi$ and all $n \geq n_{0}(\xi)$,

$$
\Psi(\lambda) \leq-\frac{1}{2 \beta}\|\lambda\|^{2}+c_{6}\|\lambda\|+\frac{1}{4 \beta}\|\lambda\|^{2}\left(1+\varepsilon_{n}\right)+f_{\mathrm{CW}}(2 \beta)
$$

with some constant $c_{6}>0$ depending on $\beta, h$ and $\|y\|$ only. Therefore, provided that $n$ is large enough to satisfy $\varepsilon_{n} \leq 1 / 3$, there exists a $\tau>1$ such that for all $\lambda$ satisfying $\|\lambda\| \geq \tau$, the right-hand side of (4.2) is bounded by $-\|\lambda\|^{2} /(8 \beta)$. Let $\tau_{p}$ denote the radius which satisfies

$$
(2 \pi)^{-p / 2} \int_{B_{\tau_{p}}(0)} \exp \left\{-\frac{1}{2}\|\lambda\|^{2}\right\} d \lambda=\frac{1}{2}
$$

By an isoperimetric inequality for Gaussian measures on $\mathbb{R}^{p}$ (cf. [11], Theorem 1.2),

$$
\left(\frac{n}{2 \pi \beta}\right)^{p / 2} \int_{B_{\tau}^{c}(0)} \exp \{n \Psi(\lambda)\} d \lambda \leq 2^{p-1} \exp \left\{-\frac{1}{2}\left[\sqrt{\frac{n}{4 \beta}} \tau-\tau_{p}\right]^{2}\right\}
$$


provided that $n$ satisfies $\sqrt{n /(4 \beta)} \tau>\tau_{p}$. Note that $\tau_{p}$ increases with the dimension $p$. Therefore, we want an upper bound for $\tau_{p}$. Since

$$
\begin{aligned}
& (2 \pi)^{-p / 2} \int_{B_{\sqrt{2 p}}^{c}(0)} \exp \left\{-\frac{1}{2}\|\lambda\|^{2}\right\} d \lambda \\
& \quad \leq(2 \pi)^{-p / 2} \exp \left\{-\frac{p}{2}\right\} \int_{\mathbb{R}^{p}} \exp \left\{-\frac{1}{4}\|\lambda\|^{2}\right\} d \lambda=\left(\frac{2}{e}\right)^{p / 2} \leq \frac{1}{2}
\end{aligned}
$$

for $p \geq 5$, it follows that

$$
\tau_{p} \leq \max \{\sqrt{2 p}, 4\} .
$$

Going back to (4.3), we find

$$
\left(\frac{n}{2 \pi \beta}\right)^{p / 2} \int_{B_{\tau}^{c}(0)} \exp \{n \Psi(\lambda)\} d \lambda \leq \exp \left\{-n \frac{\tau^{2}}{10 \beta}\right\}
$$

for large enough $n$. Next we will establish a lemma.

LEMMA 4.1. For all $r>0$,

$$
\limsup _{n \rightarrow \infty} \sup _{\lambda \in U_{r} \cap B_{\tau}(0)} \Psi(\lambda)<f_{\mathrm{CW}}(\beta, h) \quad \mathbb{P}_{\xi^{-}} \text {almost surely. }
$$

This lemma together with (4.5) shows that for $\mathbb{P}_{\xi}$-almost all $\xi$, there exist a $\delta(\xi)>0$ and an $n_{3}(\xi) \geq n_{0}(\xi)$ such that

$$
\left(\frac{n}{2 \pi \beta}\right)^{p / 2} \int_{U_{r}} \exp \{n \Psi(\lambda)\} d \lambda \leq \exp \left\{n\left[f_{\mathrm{CW}}(\beta, h)-\delta(\xi)\right]\right\}
$$

for all $n \geq n_{3}(\xi)$. The proof of Lemma 4.1 is based on the following selfaveraging property combined with a geometric argument.

LEMma 4.2. Let $f: \mathbb{R} \rightarrow \mathbb{R}$ be Lipschitz continuous. Then for all $\tau>0$ and $\mathbb{P}_{\xi}$-almost all $\xi$,

$$
\lim _{n \rightarrow \infty} \sup _{\|v\| \leq \tau}\left|\frac{1}{n} \sum_{i=1}^{n} f\left(\left\langle v, \xi_{i}\right\rangle\right)-\mathbb{E}_{\xi} f\left(\left\langle v, \xi_{1}\right\rangle\right)\right|=0
$$

PRoOF. Fix an arbitrary $\varepsilon \in(0, \tau)$ and choose $m=m(\varepsilon, \tau, p)$ points $v_{1}, \ldots, v_{m} \in B_{\tau}(0)$ such that

$$
\min _{j \in\{1, \ldots, m\}}\left\|v-v_{j}\right\| \leq \varepsilon
$$

for all $v \in \overline{B_{\tau}(0)}$. By selecting these points on a cubic lattice with spacing $\varepsilon / \sqrt{p}$, we may assume that $m \leq \exp \{p(\log (\tau / \varepsilon)+c)\}$; compare (3.6). For every $v \in \overline{B_{\tau}(0)}$ choose a $w(v) \in\left\{v_{1}, \ldots, v_{m}\right\}$ with

$$
\|v-w(v)\|=\min _{j \in\{1, \ldots, m\}}\left\|v-v_{j}\right\|
$$


Denote by $K_{f}$ the Lipschitz constant of $f$. It suffices to consider the case $K_{f}>$ 0 . Then for $\mathbb{P}_{\xi}$-almost all $\xi$, all $n \geq n_{0}(\xi)$ and all $v \in \overline{B_{\tau}(0)}$ by Proposition 2.1,

$$
\begin{aligned}
& \left|\frac{1}{n} \sum_{i=1}^{n} f\left(\left\langle v, \xi_{i}\right\rangle\right)-\frac{1}{n} \sum_{i=1}^{n} f\left(\left\langle w(v), \xi_{i}\right\rangle\right)\right| \\
& \quad \leq K_{f}\left(\frac{1}{n} \sum_{i=1}^{n}\left\langle v-w(v), \xi_{i}\right\rangle^{2}\right)^{1 / 2} \\
& \quad \leq K_{f}\left(1+\varepsilon_{n}\right)^{1 / 2}\|v-w(v)\| \leq K_{f}\left(1+\varepsilon_{n}\right)^{1 / 2} \varepsilon .
\end{aligned}
$$

Furthermore, for all $v \in \overline{B_{\tau}(0)}$,

$$
\begin{aligned}
\left|\mathbb{E}_{\xi} f\left(\left\langle v, \xi_{1}\right\rangle\right)-\mathbb{E}_{\xi} f\left(\left\langle w(v), \xi_{1}\right\rangle\right)\right| & \leq K_{f}\left(\mathbb{E}_{\xi}\left(\left\langle v-w(v), \xi_{1}\right\rangle^{2}\right)\right)^{1 / 2} \\
& =K_{f}\|v-w(v)\| \leq K_{f} \varepsilon
\end{aligned}
$$

Therefore, to prove the lemma it remains to show that

$$
\lim _{n \rightarrow \infty} \sup _{j \in\{1, \ldots, m\}}\left|\frac{1}{n} \sum_{i=1}^{n} f\left(\left\langle v_{j}, \xi_{i}\right\rangle\right)-\mathbb{E}_{\xi} f\left(\left\langle v_{j}, \xi_{1}\right\rangle\right)\right|=0
$$

$\mathbb{P}_{\xi}$-almost surely. Recall that $m$ depends on $n$ via the dimension $p$. Fix $j$ for the moment and let

$$
\begin{aligned}
A_{n, j}= & \left\{\left(\widetilde{\xi}_{1}, \ldots, \widetilde{\xi}_{n}\right) \in\{-1,1\}^{p n}:\right. \\
& \left.\left|\frac{1}{n} \sum_{i=1}^{n} f\left(\left\langle v_{j}, \widetilde{\xi}_{i}\right\rangle\right)-\mathbb{E}_{\xi} f\left(\left\langle v_{j}, \xi_{1}\right\rangle\right)\right|<\tau K_{f} \varepsilon\right\}
\end{aligned}
$$

denote the set of those realizations of the patterns for which the difference we are interested in does not exceed the bound $\tau K_{f} \varepsilon$. Then,

$$
\begin{aligned}
\mathbb{P}_{\xi}(\mid & \left.\frac{1}{n} \sum_{i=1}^{n} f\left(\left\langle v_{j}, \xi_{i}\right\rangle\right)-\mathbb{E}_{\xi} f\left(\left\langle v_{j}, \xi_{1}\right\rangle\right) \mid \geq 2 \tau K_{f} \varepsilon\right) \\
\quad & \leq \mathbb{P}_{\xi}\left(\inf _{\left(\widetilde{\xi}_{1}, \ldots, \widetilde{\xi}_{n}\right) \in A_{n, j}} \frac{1}{n} \sum_{i=1}^{n}\left|f\left(\left\langle v_{j}, \xi_{i}\right\rangle\right)-f\left(\left\langle v_{j}, \widetilde{\xi}_{i}\right\rangle\right)\right| \geq \tau K_{f} \varepsilon\right)
\end{aligned}
$$

by the definition of $A_{n, j}$. Now,

$$
\begin{gathered}
\inf _{\left(\widetilde{\xi}_{1}, \ldots, \widetilde{\xi}_{n}\right) \in A_{n, j}} \frac{1}{n} \sum_{i=1}^{n}\left|f\left(\left\langle v_{j}, \xi_{i}\right\rangle\right)-f\left(\left\langle v_{j}, \widetilde{\xi}_{i}\right\rangle\right)\right| \\
\leq \frac{K_{f}}{n} \inf _{\left(\widetilde{\xi}_{1}, \ldots, \widetilde{\xi}_{n}\right) \in A_{n, j}} \sum_{i=1}^{n} g_{v_{j}}\left(\xi_{i}, \widetilde{\xi}_{i}\right),
\end{gathered}
$$

where $g_{v_{j}}\left(\xi_{i}, \widetilde{\xi}_{i}\right)=\left|\left\langle v_{j}, \xi_{i}-\widetilde{\xi}_{i}\right\rangle\right|$ serves as a weighted distance function on the set $\{-1,1\}^{p} \times\{-1,1\}^{p}$. By [13], Theorem 2.4.1, we have for all $t>0$ the 
isoperimetric inequality

$$
\begin{aligned}
& \mathbb{E}_{\xi} \exp \left\{t \inf _{\left(\tilde{\xi}_{1}, \ldots, \widetilde{\xi}_{n}\right) \in A_{n, j}} \sum_{i=1}^{n} g_{v_{j}}\left(\xi_{i}, \widetilde{\xi}_{i}\right)\right\} \\
& \leq \frac{1}{\mathbb{P}_{\xi}\left(A_{n, j}\right)}\left(\mathbb{E}_{\xi} \cosh \left(\operatorname{tg}_{v_{j}}\left(\xi_{1}, \xi_{2}\right)\right)\right)^{n} .
\end{aligned}
$$

Calculating the expectation gives

$$
\begin{aligned}
\mathbb{E}_{\xi} \cosh \left(\operatorname{tg}_{v_{j}}\left(\xi_{1}, \xi_{2}\right)\right) & =\mathbb{E}_{\xi} \exp \left\{t\left\langle v_{j}, \xi_{1}-\xi_{2}\right\rangle\right\}=\prod_{\mu=1}^{p}\left[\frac{1}{2}\left(1+\cosh \left(2 t v_{j}^{\mu}\right)\right)\right] \\
& \leq \prod_{\mu=1}^{p} \exp \left\{2 t^{2}\left(v_{j}^{\mu}\right)^{2}\right\}=\exp \left\{2 t^{2}\left\|v_{j}\right\|^{2}\right\} \leq \exp \left\{2 t^{2} \tau^{2}\right\}
\end{aligned}
$$

and by estimating the probability of $A_{n, j}^{c}$ with the help of Chebyshev's inequality, we get

$$
\begin{aligned}
\mathbb{P}_{\xi}\left(A_{n, j}^{c}\right) & \leq\left(n \tau K_{f} \varepsilon\right)^{-2} \mathbb{E}_{\xi}\left(\left|\sum_{i=1}^{n}\left(f\left(\left\langle v_{j}, \xi_{i}\right\rangle\right)-\mathbb{E}_{\xi} f\left(\left\langle v_{j}, \xi_{1}\right\rangle\right)\right)\right|^{2}\right) \\
& \leq\left(n \tau K_{f} \varepsilon\right)^{-2} \sum_{i=1}^{n} \mathbb{E}_{\xi}\left(\left|f\left(\left\langle v_{j}, \xi_{i}\right\rangle\right)-\mathbb{E}_{\xi} f\left(\left\langle v_{j}, \xi_{1}\right\rangle\right)\right|^{2}\right) \\
& \leq n\left(n \tau K_{f} \varepsilon\right)^{-2} \mathbb{E}_{\xi}\left(\left|f\left(\left\langle v_{j}, \xi_{1}\right\rangle\right)-f(0)\right|^{2}\right) \\
& \leq\left(n \tau^{2} \varepsilon^{2}\right)^{-1} \mathbb{E}_{\xi}\left(\left\langle v_{j}, \xi_{1}\right\rangle^{2}\right) \\
& \leq \frac{1}{n \varepsilon^{2}}
\end{aligned}
$$

We will assume $n \geq 2 / \varepsilon^{2}$ to assure $\mathbb{P}_{\xi}\left(A_{n, j}\right) \geq \frac{1}{2}$. By (4.7), (4.8), another application of Chebyshev's inequality and (4.9),

$$
\mathbb{P}_{\xi}\left(\left|\frac{1}{n} \sum_{i=1}^{n} f\left(\left\langle v_{j}, \xi_{i}\right\rangle\right)-\mathbb{E}_{\xi} f\left(\left\langle v_{j}, \xi_{1}\right\rangle\right)\right| \geq 2 \tau K_{f} \varepsilon\right) \leq 2 \exp \{-n \varepsilon \tau t\} \exp \left\{2 n \tau^{2} t^{2}\right\}
$$

for all $t>0$. Choosing the optimal $t$, which is $t=\varepsilon /(4 \tau)$, yields

$$
\begin{aligned}
& \mathbb{P}_{\xi}\left(\sup _{j \in\{1, \ldots, m\}}\left|\frac{1}{n} \sum_{i=1}^{n} f\left(\left\langle v_{j}, \xi_{i}\right\rangle\right)-\mathbb{E}_{\xi} f\left(\left\langle v_{j}, \xi_{1}\right\rangle\right)\right| \geq 2 \tau K_{f} \varepsilon\right) \\
& \quad \leq 2 m \exp \left\{-n \frac{\varepsilon^{2}}{8}\right\} \leq \exp \left\{-n \frac{\varepsilon^{2}}{10}\right\}
\end{aligned}
$$

for all large enough $n$ by the bound on $m$. Now (4.6) follows from the BorelCantelli lemma. 
Proof of Lemma 4.1. Recall the definitions of $\Psi$ and $y_{n}$ from Lemma 3.3. By the self-averaging property applied to the Lipschitz continuous function $\log \cosh$,

$$
\lim _{n \rightarrow \infty} \sup _{\|\lambda\| \leq \tau}\left|\Psi(\lambda)+\frac{1}{2 \beta}\left\|\lambda-h e_{l}-y_{n}\right\|^{2}-\mathbb{E}_{\xi} \log \cosh \left\langle\lambda, \xi_{1}\right\rangle\right|=0
$$

$\mathbb{P}_{\xi}$-almost surely. Since

$$
\begin{aligned}
\left|\left\|\lambda-h e_{l}-y_{n}\right\|^{2}-\left\|\lambda-h e_{l}\right\|^{2}\right| & \leq 2\left|\left\langle\lambda-h e_{l}, y_{n}\right\rangle\right|+\left\|y_{n}\right\|^{2} \\
& \leq 2(\tau+h) \frac{\|y\|}{\sqrt{n}}+\frac{\|y\|^{2}}{n} \rightarrow 0
\end{aligned}
$$

for all $\lambda \in B_{\tau}(0)$ and $\left\|\lambda-h e_{l}\right\|^{2}=\mathbb{E}_{\xi}\left(\left\langle\lambda-h e_{l}, \xi_{1}\right\rangle^{2}\right)$ for all $\lambda \in \mathbb{R}^{p}$, it suffices to show

$$
\begin{aligned}
& \limsup _{n \rightarrow \infty} \sup _{\lambda \in U_{r} \cap B_{\tau}(0)} \mathbb{E}_{\xi}\left\{-\frac{1}{2 \beta}\left(\left\langle\lambda, \xi_{1}\right\rangle-h \xi_{1}^{l}\right)^{2}+\log \cosh \left\langle\lambda, \xi_{1}\right\rangle\right\} \\
& <f_{\mathrm{CW}}(\beta, h) .
\end{aligned}
$$

In the case $h=0$, by the definition of $z$ and $f_{\mathrm{CW}}(\beta)$ this can be done by proving the existence of a constant $\delta>0$, depending only on $r$, such that

$$
\mathbb{P}_{\xi}\left(||\left\langle\lambda, \xi_{1}\right\rangle|-| \operatorname{arctanh} z|| \geq \delta\right) \geq \frac{1}{18}
$$

for all $\lambda \in U_{r} \cap B_{\tau}(0)$ and all $p \in \mathbb{N}$. In the case $h \neq 0$, (4.10) can be established by showing

$$
\mathbb{P}_{\xi}\left(\left|\left\langle\lambda, \xi_{1}\right\rangle-\operatorname{arctanh} z \xi_{1}^{l}\right| \geq \delta\right) \geq \frac{1}{18}
$$

for all $\lambda \in U_{r} \cap B_{\tau}(0)$ and all $p \in \mathbb{N}$ with a constant $\delta>0$, depending only on $r$. We will show that $\delta=r / 42$ suffices in both cases. The case $h \neq 0$ is a direct consequence of the following result on Rademacher averages, applied with $X=\left\langle\lambda-\operatorname{arctanh} z e_{l}, \xi_{1}\right\rangle$ which satisfies $\mathbb{E}_{\xi} X^{2} \geq r^{2}$. For background on Rademacher averages, see [11], Chapter 4, for instance.

LEMMA 4.3. For every Rademacher average $X$,

$$
\mathbb{P}\left(|X| \geq \frac{1}{8} \sqrt{\mathbb{E} X^{2}}\right)>\frac{1}{3} .
$$

PRoof. By scaling we may assume that $\mathbb{E} X^{2}=1$. From the Khintchine inequality (cf. [11], Lemma 4.1), we know $\mathbb{E}|X| \geq 1 / \sqrt{2}$. Therefore, by the Schwarz inequality,

$$
\begin{aligned}
\frac{1}{\sqrt{2}} \leq \mathbb{E}|X| & \leq \frac{1}{8}+\mathbb{E}\left(|X| 1_{\{|X|>1 / 8\}}\right) \\
& \leq \frac{1}{8}+\sqrt{\mathbb{E}\left(X^{2}\right) \mathbb{P}\left(|X|>\frac{1}{8}\right)}
\end{aligned}
$$


and, therefore,

$$
\mathbb{P}\left(|X|>\frac{1}{8}\right) \geq\left(\frac{1}{\sqrt{2}}-\frac{1}{8}\right)^{2}>\frac{1}{3}
$$

To treat the case $h=0$, we fix $\lambda \in U_{r} \cap B_{\tau}(0)$. Without loss of generality, we may assume $z \geq 0$ as well as $\left|\lambda_{1}\right| \geq\left|\lambda_{\mu}\right|$ for all $\mu \in\{2, \ldots, p\}$ and $\lambda_{1} \geq 0$. In the sequel, we will use the abbreviations $\tilde{\lambda}=\left(\lambda_{2}, \ldots, \lambda_{p}\right)$ and $\eta=\left(\xi_{1}^{2}, \ldots, \xi_{1}^{p}\right)$. First, we will consider the case when $\|\tilde{\lambda}\|$ is small.

Let $\|\tilde{\lambda}\| \leq r / 3$. Then $\left|\lambda_{1}-\operatorname{arctanh} z\right|^{2}=\left\|\lambda-\operatorname{arctanh} z e_{1}\right\|^{2}-\|\tilde{\lambda}\|^{2} \geq 8 r^{2} / 9$. From the triangle inequality, we find ||$x+y|-\tilde{z}| \geq|| x|-\tilde{z}|-|y|$ for all $x$, $y \in \mathbb{R}$ and all $\tilde{z} \geq 0$. Therefore, by Chebyshev's inequality,

$$
\begin{aligned}
& \mathbb{P}_{\xi}\left(||\left\langle\lambda, \xi_{1}\right\rangle|-\operatorname{arctanh} z| \geq \frac{r}{3}\right)=\mathbb{P}_{\xi}\left(|| \lambda_{1} \xi_{1}^{1}+\langle\tilde{\lambda}, \eta\rangle|-\operatorname{arctanh} z| \geq \frac{r}{3}\right) \\
& \quad \geq \mathbb{P}_{\xi}\left(\left|\lambda_{1}-\operatorname{arctanh} z\right|-|\langle\tilde{\lambda}, \eta\rangle| \geq \frac{r}{3}\right) \\
& \quad \geq 1-\mathbb{P}_{\xi}\left(|\langle\tilde{\lambda}, \eta\rangle|>\frac{r}{2}\right) \\
& \quad \geq 1-\left(\frac{2}{r}\right)^{2} \mathbb{E}_{\xi}\left(\langle\tilde{\lambda}, \eta\rangle^{2}\right) \geq \frac{5}{9} .
\end{aligned}
$$

It remains to consider the case $\|\tilde{\lambda}\|>r / 3$. When $\left|\lambda_{1}\right| \leq r / \sqrt{27}$, then there exists a $k \in\{2, \ldots, p-2\}$ such that

$$
a=\left(\sum_{\mu=1}^{k} \lambda_{\mu}^{2}\right)^{1 / 2} \geq \frac{r}{\sqrt{27}} \text { and } b=\left(\sum_{\mu=k+1}^{p} \lambda_{\mu}^{2}\right)^{1 / 2} \geq \frac{r}{\sqrt{27}} .
$$

(Recall that $\lambda_{1} \geq\left|\lambda_{\mu}\right|$ for all $\mu \in\{2, \ldots, p\}$ by assumption.) When $\left|\lambda_{1}\right|>$ $r / \sqrt{27}$, then the same holds with $k=1$. Let

$$
X=\frac{1}{a} \sum_{\mu=1}^{k} \lambda_{\mu} \xi_{1}^{\mu} \quad \text { and } \quad Y=\frac{1}{b} \sum_{\mu=k+1}^{p} \lambda_{\mu} \xi_{1}^{\mu} .
$$

Then $\left\langle\lambda, \xi_{1}\right\rangle$ can be rewritten as $\left\langle\lambda, \xi_{1}\right\rangle=a X+b Y$ with $a, b \geq r / \sqrt{27}$ and independent Rademacher averages $X$ and $Y$, satisfying $\mathbb{E}_{\xi} X^{2}=\mathbb{E}_{\xi} Y^{2}=1$. Now, (4.11) follows from the following lemma with $\varrho=r / \sqrt{27}$, and the proof of Lemma 4.1 is complete.

Lemma 4.4. Let $\varrho>0, a, b \geq \varrho$ and $\tilde{z} \geq 0$. Consider independent Rademacher averages $X$ and $Y$, satisfying $\mathbb{E} X^{2}=\mathbb{E} Y^{2}=1$. Then

$$
\mathbb{P}(|| a X+b Y|-\tilde{z}| \geq \varrho / 8)>1 / 18 .
$$


PRoof. Let $\kappa, \tilde{\kappa}$ be two independent Bernoulli random variables, independent of $X$ and $Y$, satisfying $\mathbb{P}(\kappa= \pm 1)=\mathbb{P}(\tilde{\kappa}= \pm 1)=1 / 2$. Then $a X+b Y$ equals $a \kappa X+b \tilde{\kappa} Y$ in distribution and, therefore,

$$
\mathbb{P}\left(|| a X+b Y|-\tilde{z}| \geq \frac{\varrho}{8}\right)=\mathbb{E}\left(\mathbb{P}\left(|| a \kappa X+b \tilde{\kappa} Y|-\tilde{z}| \geq \frac{\varrho}{8} \mid X, Y\right)\right) .
$$

For the conditional probability we have the estimate

$$
\mathbb{P}\left(|| a \kappa X+b \tilde{\kappa} Y|-\tilde{z}| \geq \frac{\varrho}{8} \mid X, Y\right) \geq \frac{1}{2} 1_{\{\min \{|a X|,|b Y|\} \geq \varrho / 8\}},
$$

which can be seen as follows: assume $\min \{|a X|,|b Y|\} \geq \varrho / 8$ and suppose that ||$a X+b Y|-\tilde{z}|<\varrho / 8$. Then the triangle inequality implies

||$a X-b Y|-\tilde{z}| \geq|| a X-b Y|-| a X+b Y||-\frac{\varrho}{8}=2 \min \{|a X|,|b Y|\}-\frac{\varrho}{8} \geq \frac{\varrho}{8}$.

Therefore, at least half of the realizations of the signs $\kappa$ and $\tilde{\kappa}$ lead to the estimate ||$a \kappa X+b \tilde{\kappa} Y|-\tilde{z}| \geq \varrho / 8$. Returning to (4.12), we find

$$
\mathbb{P}\left(|| a X+b Y|-\tilde{z}| \geq \frac{\varrho}{8}\right) \geq \frac{1}{2} \mathbb{P}\left(\min \{|a X|,|b Y|\} \geq \frac{\varrho}{8}\right) .
$$

By Lemma 4.3 , we know $\mathbb{P}(a|X| \geq \varrho / 8)>1 / 3$ and $\mathbb{P}(b|Y| \geq \varrho / 8)>1 / 3$. Therefore,

$$
\mathbb{P}\left(|| a X+b Y|-\tilde{z}| \geq \frac{\varrho}{8}\right)>\frac{1}{18}
$$

Taylor expansion of $\Psi$. Before turning to the intermediate and the inner region, we need to investigate $\Psi$ given in (3.19). Since $\lambda_{\nu}^{n}(\xi)$ for $\nu \in L$ is a local maximum of $\Phi$ by Proposition 2.3(b), the Taylor expansion of $\Psi$ in $\lambda_{\nu}^{n}(\xi)$ gives

$$
\begin{aligned}
\Psi(\lambda)= & \Phi\left(\lambda_{\nu}^{n}(\xi)\right)+\left\langle x_{\nu}^{n}(\xi), y_{n}\right\rangle-\frac{1}{2 \beta}\left\|y_{n}\right\|^{2}+\frac{1}{\beta}\left\langle\lambda-\lambda_{\nu}^{n}(\xi), y_{n}\right\rangle \\
& -\frac{1}{2}\left\langle\lambda-\lambda_{\nu}^{n}(\xi), C_{\nu}^{n}(\xi)\left[\lambda-\lambda_{\nu}^{n}(\xi)\right]\right\rangle+R_{\nu}^{n}\left(\lambda-\lambda_{\nu}^{n}(\xi), \xi\right)
\end{aligned}
$$

for all $\lambda \in \mathbb{R}^{p}$ and all $n \geq n_{1}(\xi)$, where $C_{\nu}^{n}(\xi)$ is given by (3.18). The remainder $R_{\nu}^{n}$ is given by

$$
R_{\nu}^{n}(\lambda, \xi)=-\frac{1}{n} \sum_{i=1}^{n}\left(\int_{0}^{1}(1-t)^{2} \frac{\tanh }{\cosh ^{2}}\left(\left\langle\lambda_{\nu}^{n}(\xi)+t \lambda, \xi_{i}\right\rangle\right) d t\right)\left\langle\lambda, \xi_{i}\right\rangle^{3} .
$$

By Proposition 2.1 and Lemma 3.1, we have the following bound on the remainder: for arbitrary $a, \varrho>0$, both possibly depending on $n \in \mathbb{N}$, for $\mathbb{P}_{\xi}$-almost 
all $\xi$, all $n \geq n_{2}(\xi)$ and all $\lambda \in B_{\varrho}(0)$,

$$
\begin{aligned}
\left|R_{\nu}^{n}(\lambda, \xi)\right| \leq & \frac{1}{n} \sum_{i=1}^{n}\left\langle\lambda, \xi_{i}\right\rangle^{2} 1_{\left\{\left|\left\langle\lambda, \xi_{i}\right\rangle\right|>a\right\}}\left|\int_{\left\langle\lambda_{\nu}^{n}(\xi), \xi_{i}\right\rangle}^{\left\langle\lambda_{\nu}^{n}(\xi)+\lambda, \xi_{i}\right\rangle} \frac{|\tanh |}{\cosh ^{2}}(s) d s\right| \\
& +\frac{1}{n} \sum_{i=1}^{n}\left|\left\langle\lambda, \xi_{i}\right\rangle\right|^{3} 1_{\left\{\left|\left\langle\lambda, \xi_{i}\right\rangle\right| \leq a\right\}} \int_{0}^{1} \frac{|\tanh |}{\cosh ^{2}}\left(\left\langle\lambda_{\nu}^{n}(\xi)+t \lambda, \xi_{i}\right\rangle\right) d t \\
\leq & \Gamma\left(n, \frac{a}{\varrho}\right)\|\lambda\|^{2} 4 \int_{\mathbb{R}} \exp (-2|s|) d s+a \frac{1}{n} \sum_{i=1}^{n}\left\langle\lambda, \xi_{i}\right\rangle^{2} \\
\leq & {\left[4 \Gamma\left(n, \frac{a}{\varrho}\right)+a\left(1+\varepsilon_{n}\right)\right]\|\lambda\|^{2} . }
\end{aligned}
$$

In the case $\beta<\beta_{c}$ and $h=0$, we know from Proposition 2.3(b) that $\lambda_{\nu}^{n}(\xi)=0$ and can improve this bound to

$$
\left|R_{\nu}^{n}(\lambda, \xi)\right| \leq\left[4 \Gamma(n, a / \varrho)+a^{2}\left(1+\varepsilon_{n}\right)\right]\|\lambda\|^{2}
$$

by the estimate

$$
\frac{|\tanh |}{\cosh ^{2}}\left(\left\langle t \lambda, \xi_{i}\right\rangle\right) \leq\left|\left\langle\lambda, \xi_{i}\right\rangle\right|
$$

for all $t \in[0,1]$.

Intermediate region. Let $a \leq c_{1} / 16$ with $c_{1}$ from (2.5) and let $r$ be small enough and $n \geq \max \left\{n_{1}(\xi), n_{2}(\xi)\right\}$ large enough to satisfy

$$
4 \Gamma\left(n, \frac{a}{r}\right)+a\left(1+\varepsilon_{n}\right) \leq \frac{c_{1}}{8} \quad \text { and } \quad \varepsilon_{n} \leq 1
$$

Furthermore, let

$$
R>\max \left\{2\left[c_{2}+\frac{\|y\|}{c_{1} \beta}\right], \frac{5}{\sqrt{c_{1}}}\right\}
$$

with $c_{2}=2|z| / c_{1}$ as before. By the Taylor expansion (4.13), for every $\nu \in L$,

$$
\begin{aligned}
& \left(\frac{n}{2 \pi \beta}\right)^{p / 2} \int_{\left\{R \varepsilon_{n} \leq\left\|\lambda-\operatorname{arctanh} z e_{\nu}\right\|<r\right\}} \exp \{n \Psi(\lambda)\} d \lambda \\
& =\left(\frac{n}{2 \pi \beta}\right)^{p / 2} \exp \left\{n\left[\Phi\left(\lambda_{\nu}^{n}(\xi)\right)+\left\langle x_{\nu}^{n}(\xi), y_{n}\right\rangle-\frac{1}{2 \beta}\left\|y_{n}\right\|^{2}\right]\right\} \\
& \times \int_{\left\{R \varepsilon_{n} \leq\left\|\lambda-\operatorname{arctanh} z e_{\nu}\right\|<r\right\}} \exp \left\{-\frac{n}{2}\left\langle\lambda-\lambda_{\nu}^{n}(\xi), C_{\nu}^{n}(\xi)\left[\lambda-\lambda_{\nu}^{n}(\xi)\right]\right\rangle\right\} \\
& \times \exp \left\{\frac{n}{\beta}\left\langle\lambda-\lambda_{\nu}^{n}(\xi), y_{n}\right\rangle+n R_{\nu}^{n}\left(\lambda-\lambda_{\nu}^{n}(\xi), \xi\right)\right\} d \lambda .
\end{aligned}
$$

Before estimating the right-hand side of (4.16), let us remark that the following estimates will hold uniformly for $\nu \in L$. Substituting $\lambda+\lambda_{\nu}^{n}(\xi)+$ 
$(1 / \beta) C_{\nu}^{n}(\xi)^{-1} y_{n}$ for $\lambda$ and applying the bound (4.14) on the remainder, we see that the integral on the right-hand side of (4.16) is bounded above by

$$
\begin{aligned}
& \exp \left\{\frac{n}{2 \beta^{2}}\left\langle y_{n}, C_{\nu}^{n}(\xi)^{-1} y_{n}\right\rangle\right\} \\
& \quad \times \int \exp \left\{-\frac{n}{2}\left\langle\lambda, C_{\nu}^{n}(\xi) \lambda\right\rangle+n \frac{c_{1}}{8}\left\|\lambda+\frac{1}{\beta} C_{\nu}^{n}(\xi)^{-1} y_{n}\right\|^{2}\right\} d \lambda,
\end{aligned}
$$

where the domain of integration

$$
\left\{\left\|\lambda+\lambda_{\nu}^{n}(\xi)-\operatorname{arctanh} z e_{\nu}+\frac{1}{\beta} C_{\nu}^{n}(\xi)^{-1} y_{n}\right\| \geq R \varepsilon_{n}\right\}
$$

is contained in $\left\{\|\lambda\| \geq R \varepsilon_{n} / 2\right\}$, because $\left\|\lambda_{\nu}^{n}(\xi)-\operatorname{arctanh} z e_{\nu}\right\| \leq c_{2} \varepsilon_{n}$ and

$$
\left\|\frac{1}{\beta} C_{\nu}^{n}(\xi)^{-1} y_{n}\right\| \leq \frac{\|y\|}{c_{1} \beta \sqrt{n}}
$$

by Proposition 2.3(a). Again from Proposition 2.3(a), we know that

$$
\left\|C_{\nu}^{n}(\xi)-\frac{c_{1}}{2} \operatorname{Id}_{\mathbb{R}^{p}}\right\| \geq \frac{c_{1}}{2}
$$

Substituting $\lambda$ for $\sqrt{n}\left(C_{\nu}^{n}(\xi)-\left(c_{1} / 2\right) \operatorname{Id}_{\mathbb{R}^{p}}\right)^{1 / 2} \lambda$, we find that the integral in (4.17) is bounded above by

$$
\begin{aligned}
& \frac{1}{n^{p / 2}} \exp \left\{\frac{\|y\|^{2}}{4 c_{1} \beta^{2}}\right\} \operatorname{det}\left(C_{\nu}^{n}(\xi)-\frac{c_{1}}{2} \operatorname{Id}_{\mathbb{R}^{p}}\right)^{-1 / 2} \\
& \times \int_{\left\{\|\lambda\| \geq \sqrt{c_{1} / 2}(R / 2) \sqrt{n} \varepsilon_{n}\right\}} \exp \left\{-\frac{1}{2}\|\lambda\|^{2}\right\} d \lambda .
\end{aligned}
$$

Another application of Theorem 1.2 in [11] and (4.4) shows that the last integral is bounded by

$$
\begin{gathered}
\frac{1}{2}(2 \pi)^{p / 2} \exp \left\{-\frac{1}{2}\left[\sqrt{\frac{c_{1}}{2}} \frac{R}{2} \sqrt{n} \varepsilon_{n}-\tau_{p}\right]^{2}\right\} \\
\leq \frac{1}{2}(2 \pi)^{p / 2} \exp \left\{-\frac{1}{2}\left[\sqrt{\frac{c_{1}}{2}} \frac{R}{4} \sqrt{n} \varepsilon_{n}\right]^{2}\right\}
\end{gathered}
$$

where we used the fact that $R \sqrt{c_{1}} \geq 5$ implies

$$
\sqrt{\frac{c_{1}}{2}} \frac{R}{2} \sqrt{n} \varepsilon_{n} \geq 2 \tau_{p}
$$

for large enough $n$. 
Putting the above estimates together, we find that

$$
\begin{aligned}
\left(\frac{n}{2 \pi \beta}\right)^{p / 2} \int_{\left\{R \varepsilon_{n} \leq\left\|\lambda-\operatorname{arctanh} z e_{\nu}\right\|<r\right\}} \exp \{n \Psi(\lambda)\} d \lambda & \\
\leq & \frac{1}{2} \exp \left\{\frac{\|y\|^{2}}{4 c_{1} \beta^{2}}\right\} \exp \left\{-\frac{c_{1} R^{2}}{64} n \varepsilon_{n}^{2}\right\} \beta^{-p / 2} \operatorname{det}\left(C_{\nu}^{n}(\xi)-\frac{c_{1}}{2} \operatorname{Id}_{\mathbb{R}^{p}}\right)^{-1 / 2} \\
& \times \exp \left\{n\left[\Phi\left(\lambda_{\nu}^{n}(\xi)\right)+\left\langle x_{\nu}^{n}(\xi), y_{n}\right\rangle-\frac{1}{2 \beta}\left\|y_{n}\right\|^{2}+\frac{1}{2 \beta^{2}}\left\langle y_{n}, C_{\nu}^{n}(\xi)^{-1} y_{n}\right\rangle\right]\right\}
\end{aligned}
$$

By Proposition 2.3(a), the smallest eigenvalue of $C_{\nu}^{n}(\xi)$ is greater than or equal to $c_{1}$. Hence

$$
\operatorname{det}\left(C_{\nu}^{n}(\xi)-\frac{c_{1}}{2} \operatorname{Id}_{\mathbb{R}^{p}}\right) \geq \operatorname{det}\left(\frac{1}{2} C_{\nu}^{n}(\xi)\right)=2^{-p} \operatorname{det}\left(C_{\nu}^{n}(\xi)\right) .
$$

Since $c_{1} R^{2} \geq 25$ by the choice of $R$, we know that

$$
2^{p / 2-1} \exp \left\{-\frac{c_{1} R^{2}}{64} n \varepsilon_{n}^{2}\right\} \exp \left\{\frac{\|y\|^{2}}{4 c_{1} \beta^{2}}\right\}=o(1),
$$

where the error term $o(1)$ does not depend on $\nu$. Therefore,

$$
\begin{aligned}
& \left(\frac{n}{2 \pi \beta}\right)^{p / 2} \int_{\left\{R \varepsilon_{n} \leq\left\|\lambda-\operatorname{arctanh} z e_{\nu}\right\|<r\right\}} \exp \{n \Psi(\lambda)\} d \lambda \\
& \quad \leq \\
& \quad o(1) \operatorname{det}\left(\beta C_{\nu}^{n}(\xi)\right)^{-1 / 2} \exp \left\{n\left[\Phi\left(\lambda_{\nu}^{n}(\xi)\right)+\left\langle x_{\nu}^{n}(\xi), y_{n}\right\rangle\right]\right\} \\
& \quad \times \exp \left\{-\frac{n}{2 \beta}\left\langle y_{n},\left[\operatorname{Id}_{\mathbb{R}^{p}}-\frac{1}{\beta} C_{\nu}^{n}(\xi)^{-1}\right] y_{n}\right\rangle\right\}
\end{aligned}
$$

and

$$
\begin{aligned}
& \left(\frac{n}{2 \pi \beta}\right)^{p / 2} \int_{V_{r, R}} \exp \{n \Psi(\lambda)\} d \lambda \\
& \leq\left(\sum_{\nu \in L} \operatorname{det}\left(\beta C_{\nu}^{n}(\xi)\right)^{-1 / 2} \exp \left\{n\left[\Phi\left(\lambda_{\nu}^{n}(\xi)\right)+\left\langle x_{\nu}^{n}(\xi), y_{n}\right\rangle\right]\right\}\right. \\
& \left.\quad \times \exp \left\{-\frac{n}{2 \beta}\left\langle y_{n},\left[\operatorname{Id}_{\mathbb{R}^{p}}-\frac{1}{\beta} C_{\nu}^{n}(\xi)^{-1}\right] y_{n}\right\rangle\right\}\right) o(1) .
\end{aligned}
$$

This shows that the intermediate region does not contribute to the asymptotic behavior.

Inner region. First of all, let us remark that the following estimates will hold uniformly for $\nu \in L$ as the corresponding ones did in the previous case of the intermediate region. Choose $R$ as before. We will apply the bounds on the remainder of the Taylor expansion with $a$ replaced by

$$
a_{n}= \begin{cases}3 R \varepsilon_{n} \sqrt{\log p}, & \text { for unbounded } p \\ \sqrt{\varepsilon_{n}}, & \text { otherwise. }\end{cases}
$$


As an abbreviation, set

$$
\delta_{n}= \begin{cases}4 \Gamma\left(n, \frac{a_{n}}{R \varepsilon_{n}}\right)+a_{n}\left(1+\varepsilon_{n}\right), & \text { in the case } z \neq 0, \\ 4 \Gamma\left(n, \frac{a_{n}}{R \varepsilon_{n}}\right)+a_{n}^{2}\left(1+\varepsilon_{n}\right), & \text { otherwise. }\end{cases}
$$

Let $n \geq \max \left\{n_{1}(\xi), n_{2}(\xi)\right\}$ be large enough to satisfy $8 \delta_{n} \leq c_{1}$ which is possible since $\delta_{n} \rightarrow 0$. Note that $p \delta_{n}$ tends to zero in the following two cases: first, $z=0$ and $\left(p^{2} \log p\right) / n \rightarrow 0$ imply $p \delta_{n} \rightarrow 0$; second, $\left(p^{3} \log p\right) / n \rightarrow 0$ always implies $p \delta_{n} \rightarrow 0$.

As in the treatment of the intermediate region, by the Taylor expansion (4.13),

$$
\begin{aligned}
& \left(\frac{n}{2 \pi \beta}\right)^{p / 2} \int_{\left\{\left\|\lambda-\operatorname{arctanh} z e_{\nu}\right\|<R \varepsilon_{n}\right\}} \exp \{n \Psi(\lambda)\} d \lambda \\
& =\left(\frac{n}{2 \pi \beta}\right)^{p / 2} \exp \left\{n\left[\Phi\left(\lambda_{\nu}^{n}(\xi)\right)+\left\langle x_{\nu}^{n}(\xi), y_{n}\right\rangle-\frac{1}{2 \beta}\left\|y_{n}\right\|^{2}\right]\right\} \\
& \times \int_{\left\{\left\|\lambda-\operatorname{arctanh} z e_{\nu}\right\|<R \varepsilon_{n}\right\}} \exp \left\{-\frac{n}{2}\left\langle\lambda-\lambda_{\nu}^{n}(\xi), C_{\nu}^{n}(\xi)\left[\lambda-\lambda_{\nu}^{n}(\xi)\right]\right\rangle\right\} \\
& \times \exp \left\{\frac{n}{\beta}\left\langle\lambda-\lambda_{\nu}^{n}(\xi), y_{n}\right\rangle+n R_{\nu}^{n}\left(\lambda-\lambda_{\nu}^{n}(\xi), \xi\right)\right\} d \lambda .
\end{aligned}
$$

Again substituting $\lambda+\lambda_{\nu}^{n}(\xi)+(1 / \beta) C_{\nu}^{n}(\xi)^{-1} y_{n}$ for $\lambda$ and applying the bound (4.14), respectively, (4.15) on the remainder, we get the following upper, respectively, lower bound for the integral on the right-hand side of (4.20):

$$
\begin{aligned}
& \exp \left\{\frac{n}{2 \beta^{2}}\left\langle y_{n}, C_{\nu}^{n}(\xi)^{-1} y_{n}\right\rangle\right\} \\
& \quad \times \int \exp \left\{-\frac{n}{2}\left\langle\lambda, C_{\nu}^{n}(\xi) \lambda\right\rangle \pm n \delta_{n}\left\|\lambda+\frac{1}{\beta} C_{\nu}^{n}(\xi)^{-1} y_{n}\right\|^{2}\right\} d \lambda,
\end{aligned}
$$

where the corresponding domain of integration is

$$
\left\{\left\|\lambda+\lambda_{\nu}^{n}(\xi)-\operatorname{arctanh} z e_{\nu}+\frac{1}{\beta} C_{\nu}^{n}(\xi)^{-1} y_{n}\right\|<R \varepsilon_{n}\right\} .
$$

By the choice of $R$, it contains $\left\{\|\lambda\|<R \varepsilon_{n} / 2\right\}$. Replacing $\sqrt{n}\left(C_{\nu}^{n}(\xi) \mp\right.$ $\left.4 \delta_{n} \operatorname{Id}_{\mathbb{R}^{p}}\right)^{1 / 2} \lambda$ by $\lambda$ shows that the integral in (4.21) is bounded above, respectively, below by

$$
\begin{aligned}
& \frac{1}{n^{p / 2}} \exp \left\{ \pm 2 n \delta_{n}\left\|\frac{1}{\beta} C_{\nu}^{n}(\xi)^{-1} y_{n}\right\|^{2}\right\} \operatorname{det}\left(C_{\nu}^{n}(\xi) \mp 4 \delta_{n} \operatorname{Id}_{\mathbb{R}^{p}}\right)^{-1 / 2} \\
& \quad \times\left[\int_{\mathbb{R}^{p}} \exp \left\{-\frac{1}{2}\|\lambda\|^{2}\right\} d \lambda \pm \int_{\left\{\|\lambda\| \geq \sqrt{c_{1} / 2}(R / 2) \sqrt{n} \varepsilon_{n}\right\}} \exp \left\{-\frac{1}{2}\|\lambda\|^{2}\right\} d \lambda\right] .
\end{aligned}
$$


By (4.18) and the bound (4.19) on the second integral in (4.22), for large enough $n$ the expression (4.22) is bounded above, respectively, below by

$e^{o(1)}\left(\frac{2 \pi}{n}\right)^{p / 2}\left(\operatorname{det}\left(C_{\nu}^{n}(\xi)\right) \operatorname{det}\left(\operatorname{Id}_{\mathbb{R}^{p}} \mp 4 \delta_{n} C_{\nu}^{n}(\xi)^{-1}\right)\right)^{-1 / 2}\left[1 \pm \frac{1}{2} \exp \left[-\frac{c_{1} R^{2}}{64} n \varepsilon_{n}^{2}\right\}\right]$.

Since the smallest eigenvalue of $C_{\nu}^{n}(\xi)$ is bounded below by $c_{1}$,

$$
\left(1-\frac{4 \delta_{n}}{c_{1}}\right)^{p} \leq \operatorname{det}\left(\operatorname{Id}_{\mathbb{R}^{p}} \pm 4 \delta_{n} C_{\nu}^{n}(\xi)^{-1}\right) \leq\left(1+\frac{4 \delta_{n}}{c_{1}}\right)^{p}
$$

Hence,

$$
\begin{aligned}
& \left(\frac{n}{2 \pi \beta}\right)^{p / 2} \int_{W_{R}} \exp \{n \Psi(\lambda)\} d \lambda \\
& =\left(\sum_{\nu \in L} \operatorname{det}\left(\beta C_{\nu}^{n}(\xi)\right)^{-1 / 2} \exp \left\{n\left[\Phi\left(\lambda_{\nu}^{n}(\xi)\right)+\left\langle x_{\nu}^{n}(\xi), y_{n}\right\rangle\right]\right\}\right. \\
& \left.\quad \times \exp \left\{-\frac{n}{2 \beta}\left\langle y_{n},\left[\operatorname{Id}_{\mathbb{R}^{p}}-\frac{1}{\beta} C_{\nu}^{n}(\xi)^{-1}\right] y_{n}\right)\right\}\right) \\
& \quad \times[1+o(1)] \exp \left\{\mathscr{O}\left(p \delta_{n}\right)\right\},
\end{aligned}
$$

and the proof of Lemma 3.3 is complete.

Acknowledgments. First of all, I am grateful to Professor E. Bolthausen, who brought the problem to my attention. He gave helpful advice and constant encouragement during my work on this note as he did during my work on [8]. Warm thanks are due Dr. A. Bovier for keeping me informed about the progress of the work on [4] as well as for stimulating discussions about the central limit theorem for the overlap. In addition, I would like to thank Dr. U. Schmock for fruitful discussions and helpful comments on the present note.

\section{REFERENCES}

[1] Amit, D. J., Gutfreund, H. and Sompolinsky, H. (1985). Spin-glass models of neural networks. Phys. Rev. A 32 1007-1018.

[2] Bovier, A., Gayrard, V. and Picco, P. (1994). Gibbs states of the Hopfield model in the regime of perfect memory. Probab. Theory Related Fields 100, 329-363.

[3] Bovier, A. and GAYRARD, V. (1996). An almost sure large deviation principle for the Hopfield model. Ann. Probab. 24 1444-1475.

[4] Bovier, A. and Gayrard, V. (1996). The retrieval phase of the Hopfield model: a rigorous analysis of the overlap distribution. Probab. Theory Related Fields. To appear.

[5] Ellis, R. S. (1985). Entropy, Large Deviations, and Statistical Mechanics. Springer, New York.

[6] Figotin, A. L. and PASTuR, L. A. (1977). Exactly soluble model of a spin glass. Sov. J. Low Temp. Phys. 3 378-383.

[7] Figotin, A. L. and Pastur, L. A. (1978). Theory of disordered spin systems. Theoret. and Math. Phys. 35 403-414.

[8] Gentz, B. (1996). An almost sure central limit theorem for the overlap parameters in the Hopfield model. Stochast. Proc. Appl. 62 243-262. 
[9] Hertz, J., Krogh, A. and Palmer, R. G. (1991). Introduction to the Theory of Neural Computation. Addison-Wesley, Reading, MA.

[10] HopFIELD, J. J. (1982). Neural networks and physical systems with emergent collective computational abilities. Proc. Nat. Acad. Sci. U.S.A. 79 2554-2558.

[11] Ledoux, M. and Talagrand, M. (1991). Probability in Banach Spaces. Springer, Berlin.

[12] Martin-LöF, A. (1973). Mixing properties, differentiability of the free energy and the central limit theorem for a pure phase in the Ising model at low temperature. Comm. Math. Phys. 32 75-92.

[13] TALAgRand, M. (1995). Concentration of measure and isoperimetric inequalities in product spaces. Publ. Math. IHES 81 73-205.

\author{
ANGEWANDTE MATHEMATIK \\ UNIVERSITÄT ZÜRICH \\ WINTERTHURER STRAßE 190 \\ CH-8057 ZÜRICH \\ SWITZERLAND \\ E-MAIL: gentz@amath.unizh.ch
}

